\title{
Localized Ridge Wrinkling of Stiff Films on Compliant Substrates
}

\section{Citation}

Zang, Jianfeng, Xuanhe Zhao, Yanping Cao, and John W. Hutchinson. 2012. Localized ridge wrinkling of stiff films on compliant substrates. Journal of the Mechanics and Physics of Solids 60(7): 1265-1279.

\section{Published Version}

doi:10.1016/j.jmps.2012.03.009

\section{Permanent link}

http://nrs.harvard.edu/urn-3:HUL.InstRepos:10196728

\section{Terms of Use}

This article was downloaded from Harvard University's DASH repository, and is made available under the terms and conditions applicable to Open Access Policy Articles, as set forth at http:// nrs.harvard.edu/urn-3:HUL.InstRepos:dash.current.terms-of-use\#OAP

\section{Share Your Story}

The Harvard community has made this article openly available.

Please share how this access benefits you. Submit a story.

Accessibility 


\title{
Localized Ridge Wrinkling of Stiff Films on Compliant Substrates
}

\author{
Jianfeng Zang ${ }^{1}$, Xuanhe Zhao ${ }^{1}$, Yanping Cao ${ }^{2} *$ \& John W. Hutchinson ${ }^{3} *$ \\ ${ }^{1}$ Department of Mechanical Engineering and Material Science \\ Duke University, Durham, NC 27708, USA \\ ${ }^{2}$ AML, Department of Engineering Mechanics \\ Tsinghua University, 100084, Beijing, P. R. China \\ ${ }^{3}$ School of Engineering and Applied Sciences \\ Harvard University, Cambridge, MA 02138, USA
}

\begin{abstract}
Wrinkling of thin stiff films on thick compliant elastomeric substrates subject to plane strain compression is considered for cases in which the substrate is pre-stretched prior to film attachment. Advanced wrinkling modes are investigated that evolve as the systems are compressed beyond the onset of the primary sinusoidal wrinkling mode. If the substrate pre-stretch is greater than about $40 \%$, an advanced mode in the form of a series of well-spaced ridges separated by relatively flat film is observed in the simulations. Our experiments reveal a localization mode in the form of alternating packets of large and small amplitude wrinkles, but not ridges, while ridge formation has been observed in other recent experiments. Measurements of undulation amplitudes have been made for wrinkle fields of stiff films formed by oxidation of the surface of pre-stretched PDMS substrates. Simulations have been performed with a finite element model and an analytical film/substrate model. The formation of the ridge mode is a consequence of the altered nonlinearity of the substrate produced by the pre-stretch. The role of the tangential substrate stiffness in suppressing localization at the ridges is also highlighted. If there is no substrate pre-stretch, or if the substrate is pre-compressed, the primary sinusoidal mode gives way to an entirely different sequence of advanced modes usually entailing period doubling followed by folding. The nature of substrate nonlinearity that leads to ridges or folds is discussed.
\end{abstract}

Keywords: Wrinkling, thin films, elastomeric substrates, folds, ridges

* Correspondence may be addressed to caoyanping@tsinghua.edu.cn (Y. P. Cao) or jhutchin@fas.harvard.edu (J. W. Hutchinson). 


\section{Introduction}

Surface wrinkling of composite systems consisting of a hard skin on a soft underlayer has received considerable attention for several decades. Early studies on this issue date back to Allen (1969) with focus on the prevention of instability of layered engineering structures such as sandwich panels. Studies in recent years have demonstrated that surface wrinkling phenomenon of film/substrate systems has wide application ranging from fabricating surfaces with controlled patterns with unique wetting, optical and acoustic properties, and for measuring the mechanical properties of materials to the design of flexible electronics (Bowden et al., 1998; Stafford et al., 2004; Lacour et al.; Khang et al., 2006). Although theoretical studies during past years have led to procedures to determine the critical condition of instability and the corresponding wrinkling patterns (Allen, 1969; Volynskii et al., 2000; Groenewold, 2001; Huang and Suo, 2002; Huang et al., 2005; Huang 2005), the post-buckling evolution of surface wrinkles is only recently being pursued (Brau et al., 2010; Kim et al., 2011; Sun et al., 2012; Cao \& Hutchinson, 2012a).

This study is concerned with an unusual advanced wrinkling mode as shown in Fig. 1. Termed the mountain ridge mode, or, more briefly, the ridge mode, it was discovered through numerical simulation of wrinkling of thin stiff films on deep compliant neoHookean substrates (Cao \& Hutchinson, 2012a). The ridge mode formed when the substrate was pre-stretched in plane strain to a stretch greater than about $\lambda_{1}^{S}=1.4$ prior to film attachment (Fig. 1a). When this film/substrate system is then subject to incremental plane strain compression, the onset of wrinkling occurs as the classical sinusoidal mode at very small incremental compressive strain, typically on the order of 0.01 or less. The amplitude of the sinusoidal mode grows stably as the compression is further increased until the sinusoidal mode gives way to ridge formation at an incremental compression that is still relatively small. The ridges revert back to the periodic sinusoidal-like mode with even further compression, as will be discussed later.

This paper explores the ridge mode in greater depth. Experiments have been performed and reported in Section 2 in which localization, but not ridge formation, is observed and measured for stiff silica-like films grown on pre-stretched PDMS substrates. Other recent experiments where ridges have been observed will also be 
discussed. Numerical simulations are presented in Section 3 revealing details of ridge mode formation, including the localization aspect of the phenomenon wherein the wrinkles between the ridges decay at the expense of ridge growth. The connection between substrate nonlinearity, pre-stretch and mode-type (e.g. period-doubling and folding versus ridging) is established. A film/substrate model is proposed and analyzed in Section 4 that accounts for both the nonlinear normal traction-displacement behavior of the substrate and its tangential stiffness. The model reveals that the tangential substrate stiffness plays an essential role in formation of the advanced modes through its constraining effect on the lateral motion of the film.

\section{Experimental observations of the ridge mode}

Polydimethylsiloxane (PDMS) used for the substrate was prepared by mixing a degassed elastomer base and a crosslinker in a ratio of 10:1 w/w (Sylgard 184, Dow Corning). The pre-polymerized mixture was cast on a flat surface (a petri dish bottom or cover), and cured at $60^{\circ} \mathrm{C}$ for 6 hours. Uniaxial tensile stress-strain data for the PDMS is presented in Fig. 2. The material is modeled as being incompressible, and its ground state shear modulus is $0.32 \mathrm{MPa}$. Included in Fig. 2 are the tensile stress-strain curves for a neo-Hookean material with the same initial shear modulus and that for an ArrudaBoyce material (Arruda and Boyce, 1993), with the same ground state shear modulus and its locking parameter set at $\lambda_{m}=1.17$. These two material models will be used in the numerical simulations.

In preparation for the wrinkling tests, the cured PDMS was cut into pieces (ca. $25 \times 25 \times 1 \mathrm{~mm}$ ) and gently peeled off the casting surface. The slab of PDMS was fixed on a specially designed stretcher of in-plane dimensions, $D_{1} \times D_{3}$, where $D_{1}$ in the stretching direction is much less than $D_{3}$ in the perpendicular direction. The constraint in the perpendicular direction gives rise to desired plane strain deformations in the plane consisting of the stretch direction and the through-thickness direction of the slab. A series of slabs of PDMS fixed to the stretcher were subject to one of five pre-stretches

( $\lambda_{1}^{s}=1.2,1.3,1.35,1.4 \& 1.5$ ). Then, the pre-stretched PDMS surface was treated using RF oxygen plasma (Emitech K-1050X) at $100 W$ for $60 \mathrm{~s}$. A silica-like stiff film forms 
on PDMS surface during plasma treatment with a thickness of 10-20 nm (Befahy et al, 2010) and a Young's modulus that is approximately 1.5GPa .

The film/substrate system is then subject to increments of plane strain compression by decreasing the stretch imposed by the stretcher (cf. the depiction in Fig. 1a). After wrinkles form, the wrinkled surface was recorded by atomic force microscope (AFM) at suitable field scales (from 5 to $20 \mu \mathrm{m}$ ) at several subsequent compressive strains for each pre-stretch. A section profile across the wrinkle field was conducted for each AFM image to give the amplitude variation.

The results of these measurements are assembled in Fig. 3. Throughout this paper, the overall incremental compressive strain is defined as a nominal strain, $\varepsilon_{0}=-\left(\Delta \lambda_{1}-1\right)$, where $\Delta \lambda_{1}$ is the stretch measured from the pre-deformed state in which the film is grown. For each compressive strain, the figures display both a direct image of the wrinkled surface and an atomic force microscope (AFM) trace across the wrinkle field giving the amplitude variation. In all cases shown, the wavelength of the undulations is between 0.3 and $0.5 \mu \mathrm{m}$. The amplitude of the undulations depends on the applied compression and the form of the mode, but in all the cases shown it falls between 10 and 50 nanometers. The slopes of the undulations are therefore relatively shallow, not exceeding about $1 / 10$. While there are irregularities such as 'undulation dislocations' in the wrinkle patterns for pre-stretches at or below $\lambda_{1}^{S}=1.30$, there are no signs of undulation localization. Some evidence of localized behavior can be seen at $\lambda_{1}^{S}=1.35$, but systematic localization of the undulations is only clearly evident for the two largest pre-stretches, $\lambda_{1}^{S}=1.4 \& 1.5$. For these cases, a series of two or three large amplitude undulations is separated by multiple undulations having significantly reduced amplitudes. This reflects localization but not ridge formation. The applied compressive strain at which localization occurs is relatively small (0.043 in Fig. 3d and 0.02 in Fig. 3e). As the compressive strain is further increased the difference in amplitudes of undulations within the localized region and the undulations separating them diminishes until the mode appears to revert back to the periodic to the sinusoidal mode. This behavior is seen in Fig. $3 \mathrm{~d}$ as the compressive strain increases from 0.043 to 0.086 and even more clearly in Fig. 3e, for the compressive strain increasing from 0.02 to 0.06 . 
Based on the behavior seen in Figs. 3, it would appear that well defined localizations only form for films attached to substrates pre-stretched above (approximately) $\lambda_{1}^{S}=1.40$. The localized mode occurs as the system is compressed to strains several times greater than the strain associated with the onset of the primary sinusoidal wrinkle mode. The fully developed localization mode has multiple large undulations separated by minor undulations with amplitudes that are much smaller. Moreover, the amplitudes of minor undulations are smaller than the amplitude of the sinusoidal wrinkles at compressive strains below the onset of localization. It is evident that the large undulations gain their height at the expense of the undulations separating them-it is this feature that marks this behavior as a localization phenomenon. Lastly, it would appear that the localization mode only persists for a relatively small range of compressive strain, transitioning back to a sinusoidal-like mode as the compression increases.

The experiments in this paper reveal localization but not ridge formation. To our knowledge the only experimental evidence for localized ridge formation is the recent work of Ebata, Croll \& Crosby (2012). These authors attached films of polystyrene (with thicknesses varying from 5 to $180 \mathrm{~nm}$ ) to pre-stretched PDMS substrates. The sequence of wrinkle phenomena as the film/substrate system underwent incremental compression was measured and localized ridge formation (termed a fold with an outward morphology) was observed at compressive strains on the order of $5 \%$. The substrate pre-stretch in these experiments was considerably less than $40 \%$, in conflict with the simulations discussed next for which clear cut ridges do not form for pre-stretches less than $40 \%$.

\section{Finite element simulations of the ridge mode and other advanced modes}

Plane strain finite element simulations of film-substrate systems with substrate pre-stretch have been carried out with the aim of revealing the ridge formation phenomenon and providing insights into when it should be expected to occur. Simulations have been performed using both the neo-Hookean and the Arruda-Boyce constitutive models for elastomers. The simulations are made with the commercial software, ABAQUS [2008], and employing the hybrid element, CPE8MH, designed for simulations of incompressible materials. A detailed description of important aspects of 
the simulations was given by Cao and Hutchinson (2012a); these include the inclusion of very small initial geometric surface imperfections to trigger bifurcation modes, and the method for introduction of the pre-stretch, $\lambda_{1}^{s}$. To track the post-buckling evolution, a pseudo dynamic method (a stabilized nonlinear solution method in ABAQUS, 2008) has been adopted, which shares the similar idea with the Tikhonov regularization method (Tikhonov \& Arsenin 1977) dealing with ill-posed inverse problems, as discussed in our recent study (Cao and Hutchinson, 2012b).

Displacement-controlled loading is employed with the horizontal displacement, $u_{1}$ (taken to be independent of the vertical coordinate $x_{2}$ ), and zero shear traction specified on the vertical sides of the model. The overall compressive strain applied to the system after the film is attached to the substrate is denoted by, $\varepsilon_{0}$. It is defined as the nominal compressive strain introduced earlier as the difference, $\Delta u_{1}$, between $u_{1}$ on the two sides of the model, $\varepsilon_{0}=-\Delta u_{1} / \bar{W}$, where $\bar{W}$ is the distance between the sides in the pre-deformed state. On the bottom surface of the substrate, the vertical displacement, $u_{2}$, and the shear traction are taken to be zero. The width of the model is taken to be or the order of 10 wavelengths of the sinusoidal wrinkling mode. The depth of the substrate is taken to be more than 10 times the sinusoidal wavelength and, thus, sufficiently deep to ensure that there is no interaction with the modes and the bottom of the substrate. The substrate is effectively infinitely deep.

\subsection{Examples of the ridge mode}

Fig. 1, reproduced from Cao and Hutchinson (2012a), illustrates the evolution of the ridge mode as the system undergoes increasing compression beyond the onset of the classical sinusoidal wrinkling mode for a substrate that has been pre-stretched to $\lambda_{1}^{S}=2$. The simulations in Fig. 1 take both the film and substrate to be neo-Hookean with ground state shear modulus of the film, $\mu_{f}$, and that of the substrate, $\mu_{S}$, with ratio $\mu_{f} / \mu_{s}=836$. The primary sinusoidal mode remains stable until compressive strains more than 3 times the bifurcation strain associated with the sinusoidal mode have been imposed, at which point ridges begin to form. 
Ridges can only form at the expense of neighboring undulations in the sense that the extra length of film needed to grow the ridge feeds towards the ridge from the film on either side of the peak. This requires the film to displace tangentially towards the peak, as will be illustrated more clearly in the next section. The localization process is also evident in Fig. 4 where the height of a ridge as it emerges is compared with the height of the nearest undulation. Before the ridge begins to form, the amplitudes of the two neighboring undulations increase together. Once ridge formation begins, the ridge height increases sharply while the neighboring undulation flattens. This transition occurs over a small range of compressive strain.

Ridge formation depends on the nonlinearity of the traction-deflection behavior of the substrate, and, in particular, how this nonlinearity is affected by pre-stretch. This assertion will be developed in the sub-section which follows. However, ridge formation does not appear to be strongly dependent on the choice of elastomeric constitutive model. The sequence of wrinkle patterns in Fig. 5 has been generated using the Arruda-Boyce (1993) constitutive model with parameters chosen to fit the tensile stress-strain data for PDMS (see Fig. 2). The emergence of the ridge mode for this constitutive model is similar to that that seen for the neo-Hookean system in Fig. 1. In the simulation shown in Fig. 5 the compression is increased to a strain well beyond the onset of the ridge formation. At compressive strains above $\varepsilon=0.2$ the amplitudes of ridges have decreased and the localizations revert to the uniform sinusoidal-like undulations. In each of the simulations in Fig.1 and in Fig. 5, the same material model was used to represent the film as the substrate but with a very large stiffness relative to the substrate. Because the film is stiff, the strains in the film remain small, well within range such that the response of the material model is linear. In other words, effectively, the film is represented as being an incompressible linear elastic material.

The minimum pre-stretch required to produce the ridge mode was found to be approximately $\lambda_{1}^{S}=1.4$ for the Arruda-Boyce material with the locking parameter set at $\lambda_{m}=1.17$. This is in reasonable accord with the experimental observations reported in Section 2 which gave an indication of weak localization at $\lambda_{1}^{S}=1.35$ and well developed localization at $\lambda_{1}^{S}=1.40$ and above. For larger values of the locking parameter, the 
mimimum required pre-stretch is somewhat higher, $\lambda_{1}^{S} \cong 1.5$, and similar to that for the neo-Hookean material. The minimum pre-stretch required to produce the ridge mode is not strongly dependent on $\mu_{f} / \mu_{s}$. In addition, for a neo-Hookean substrate prestretched to $\lambda_{1}^{S}=2$, all film/substrate combinations with $\mu_{f} / \mu_{S}>10$ were found to undergo ridge localization (Cao \& Hutchinson, 2012a).

The effect of a pre-compression of the substrate, $\lambda_{0}^{S}=0.7$, on advanced mode formation is illustrated in Fig. 6 for the case of a substrate described by the Arruda-Boyce constitutive law fit to the PDMS and a film/substrate modulus ratio, $\mu_{f} / \mu_{S}=100$. Under pre-compression, or with no pre-deformation at all, ridges do not form. Instead, period-doubling occurs as the system is compressed beyond the onset of the sinusoidal mode. As more compression is applied, folds emerge in the form of sharp inward deflections. The folds grow at the expense of the intermediate undulations which become almost flat. Period-doubling and folding has been documented thoroughly through experiments by Brau et al. (2010) and through experiments and simulations by Sun, et al. (2012). Further simulations over wide range of the film/substrate modulus ratio have been presented by Cao and Hutchinson (2012a). Given the previous studies of perioddoubling and folding in the literature and the emphasis here on the ridge mode, no further examples involving pre-compression will be presented here. Nevertheless, it should be mentioned that a quantitative connection between the onset of period-doubling and precompression has not been established.

\subsection{Effect of pre-deformation on nonlinearity of substrate traction-displacement behavior}

Two subsidiary calculations have been carried out to give further insight into the role of substrate pre-stretch or pre-compression underlying the formation of the two distinct advanced modes, ridges and folds. These calculations help to expose the nature of the nonlinearity of a semi-infinite neo-Hookean substrate as dependent on a uniform pre-compression or pre-stretch, $\lambda_{1}^{S}$.

The first imposes incremental nominal tractions, $\left(s_{22}, s_{21}\right)$, on the surface of a substrate subject to uniform plane strain pre-deformation, $\lambda_{1}^{S}$. The specified tractions are 


$$
s_{22}=p_{0} \cos (2 \pi X / L), s_{21}=0
$$

where $X$ is the horizontal coordinate in the surface in the pre-deformed state, $s_{22}$ (per area in the pre-deformed state) at $X=0$ is $s_{22}=p_{0}$, and, here, $L$ is an arbitrary scaling length. The vertical displacement has the form $u_{2} / L=f\left(p_{0} / \mu_{S}, X / L\right)$ where $f$ is dimensionless with period $X / L=1$. The dependence of $\xi \equiv u_{2} / L$ on $s_{22} / \mu_{S}$ at $X=0$ is displayed in Fig. 7. Note that at $X=0, \xi>0$ corresponds to a crest and $\xi<0$ to a valley, and the nonlinearity affects the development of a crest relative to a valley. The findings related to crest-valley deformations at $X=0$ are summarized as follows:

(a) No pre-stretch $\left(\lambda_{1}^{S}=1\right)$; approx. linear: $|\xi| \leq .15$

(b) Pre-stretch $\left(\lambda_{1}^{S}=2\right)$; softening: $0<\xi<.1$, hardening: $-.1<\xi<0$

(c) Pre-compression $\left(\lambda_{1}^{S}=0.7\right)$; hardening: $0<\xi<.15$, approx. linear: $-.15<\xi<0$ Pre-stretch produces softening for outward displacements and hardening for inward displacements which favors ridge formation. Conversely, pre-compression leads to significant hardening for outward displacements and a near-linear response for $-.15<\xi<0$, and this certainly discourages ridge formation. The role of pre-compression in favoring folds is further revealed by the second calculation presented below. The case of no pre-stretch would appear to be neutral. In fact, period-doubling and folding do not occur in systems with no pre-stretch until the system has been compressed to a strain which is typically $\varepsilon_{0} \cong 0.2$ (Cao and Hutchinson, 2012a).

The second subsidiary calculation imposes a localized ridge-like or fold-like deformation on the surface of the uniformly deformed substrate and evaluates the energy required to create it. The displacements imposed on the surface are

$$
u_{1}=0 \text { and } u_{2}=4 \xi L e^{-(X / L)^{2}}
$$

with $\xi>0$ corresponding to a ridge and $\xi<0$ to a fold. Denote the work done to impose the displacements (3.2) by $\Delta \Xi$. Equivalently, this is the change in elastic energy in the substrate. When the half-space has been subject to a pre-stretch, $\lambda_{0}^{S}=2$, the ratio of the energy to create a fold-like deformation to that to create a ridge-like deformation (both with amplitude $|\xi|=0.4$ ) is 


$$
\frac{(\Delta \Xi)_{\xi=-0.4}}{(\Delta \Xi)_{\xi=+0.4}}=1.13
$$

When the substrate is subject to a pre-compression, $\lambda_{0}^{S}=0.6$, the same ratio is

$$
\frac{(\Delta \Xi)_{\xi=-0.4}}{(\Delta \Xi)_{\xi=+0.4}}=0.92
$$

These results, like those for the sinusoidal surface loading apply for any length $L$. Both sets of results suggest the following: a pre-stretch favors the occurrence of outward deflections of the surface (e.g., ridges) while pre-compression favors inward deflections (e.g., folds).

\section{An ordinary differential equation model for the nonlinear wrinkling behavior of a stiff film on a compliant substrate}

The following one-dimensional model represents the stiff film bonded to a substrate by a one-dimensional nonlinear von Karman plate subject to normal and tangential tractions that are intended to approximate the substrate constraint in its current state of pre-stretch. The vertical and horizontal displacements of the plate middle surface are denoted by $W$ and $U$; these are functions of the horizontal coordinate $X$. The strains in a von Karman plate are considered small but rotations can be moderately large, i.e., $(d W / d X)^{2}<<1$. A uniform horizontal compression, $-\varepsilon_{0}$, with associated horizontal displacement, $U_{0}(X)=-\varepsilon_{0} X$, is imposed on the system and this state is taken as the reference. The film middle surface in this uniform state is chosen to coincide with $W=0$, and the change of horizontal displacement from this state is denoted by $\Delta U=U-U_{0}$. The normal component of traction exerted on the plate by the substrate (acting downward) is taken to be nonlinear in the vertical displacement of the plate middle surface, $W(X)$ :

$$
T_{2}=K_{2} W+K_{22} W^{2}+K_{23} W^{3}
$$

The tangential traction exerted on the plate by the substrate (acting leftward) is taken to be linear in the horizontal displacement of the middle surface from the reference state:

$$
T_{1}=K_{1} \Delta U
$$


The film's moment/length, $M$, and resultant stress, $N$, are given in terms of the bending strain, $K=d^{2} W / d X^{2}$, and the stretching strain, $\varepsilon=d U / d X+\frac{1}{2}(d W / d X)^{2}$, by

$$
M=\frac{\bar{E} h^{2}}{12} K \text { and } N=\bar{E} h \varepsilon
$$

with $E$ and $v$ as the film Young's modulus and Poisson's ratio, $\bar{E}=E$ / $\left(1-v^{2}\right)$ and $h$ as the film thickness. The principle of virtual work for von Karman plate theory gives the equilibrium equations:

$$
\frac{d^{2} M}{d^{2} X}-\frac{d}{d X}\left(N \frac{d W}{d X}\right)=-T_{2} \text { and } \quad \frac{d N}{d X}=T_{1}
$$

Bifurcation from the uniform state is governed by the linearized equation:

$$
\frac{\bar{E} h^{3}}{12} \frac{d^{4} W}{d^{4} X}+\bar{E} h \varepsilon_{0} \frac{d^{2} W}{d^{2} X}+K_{2} W=0
$$

The critical eigenvalue, eigenmode and wavelength for an unbounded film are

$$
\varepsilon_{0}^{C}=\sqrt{\frac{K_{2} h}{3 \bar{E}}}, \quad W=\cos \left(\frac{2 \pi X}{L}\right) \text { with } \frac{L}{h}=2 \pi\left(\frac{\bar{E}}{12 K_{2} h}\right)^{1 / 4}
$$

The eigenmodal tangential displacement, $\Delta U$, is zero.

The nonlinear system of equations is now put into dimensionless form. This step is important because it permits realistic choices to be made for the coefficients in the traction-displacement relations representing the substrate. Noting (4.6), define a length quantity proportional wavelength of the critical mode by $d=h\left(\bar{E} / K_{2} h\right)^{1 / 4}$, and let $x=X / d$. Further, let $w=W / h, u=U d / h^{2}, \Delta u=\Delta U d / h^{2}, n=N d^{2} / \bar{E} h^{3}$, $e=\varepsilon d^{2} / h^{2}, t_{2}=T_{2} d^{4} / \bar{E} h^{4}$ and $t_{1}=T_{1} d^{3} / \bar{E} h^{3}$. The resulting system of dimensionless, nonlinear equations is

$$
\begin{aligned}
& \frac{1}{12} w^{\prime \prime \prime}-\left(n w^{\prime}\right)^{\prime}=-t_{2}, \quad t_{2}=w+k_{22} w^{2}+k_{23} w^{3} \\
& n^{\prime}=t_{1}, \quad t_{1}=k_{1} \Delta u
\end{aligned}
$$

\footnotetext{
${ }^{1}$ In this model, the middle surface of the film is taken to lie along the top surface of the substrate. A more accurate description joins the film and substrate along the bottom surface of the film (Cai et al., 2011). The difference in predictions from the two formulations is small and unimportant for present purposes.
} 


$$
n=-e_{0}+\Delta u^{\prime}+\frac{1}{2} w^{\prime 2}
$$

with ( $)^{\prime}=d\left(\mathrm{)} / d x, e_{0}=\varepsilon_{0} d^{2} / h^{2}\right.$, and with dimensionless traction coefficients

$$
k_{1}=K_{1} h^{1 / 2} /\left(\bar{E} K_{2}\right)^{1 / 2}, k_{22}=K_{22} h / K_{2} \text { and } k_{23}=K_{23} h^{2} / K_{2}
$$

Loading is prescribed by imposing the dimensionless overall compressive strain, $e_{0}$.

For periodic solutions with wavelength, $X_{0}$, the energy in the system (per out-ofplane length), $\Pi$, evaluated over one wavelength, is

$$
\Pi /\left(K_{2} h^{2} d\right)=\int_{0}^{x_{0}}\left\{\frac{1}{24} w^{\prime \prime 2}+\frac{1}{2} n^{2}+\frac{1}{2} k_{1} \Delta u^{2}+\frac{1}{2} w^{2}+\frac{1}{3} k_{22} w^{3}+\frac{1}{4} k_{23} w^{4}\right\} d x
$$

with $n$ given by (4.7c) and $x_{0}=X_{0} / d$. The Euler-Lagrange equations derived from the first variations of (4.8) with respect to $w$ and $\Delta u$ are (4.7a,b).

The dimensionless tangential traction coefficient $k_{1}$ plays an important role in the advanced post-bifurcation modes and especially in localization. To obtain a sense of its magnitude, consider sinusoidal tractions, $\left(T_{1} \sin (2 \pi X / L), T_{2} \cos (2 \pi X / L)\right)$, applied to the surface of a unstretched, semi-infinite neo-Hookean substrate with shear modulus $\mu_{s}$. The linearized surface displacements are $(\Delta U \sin (2 \pi X / L), W \cos (2 \pi X / L))$ where

$$
T_{1}=\frac{4 \pi \mu_{S}}{L} \Delta U \equiv K_{1} \Delta U, \quad T_{2}=\frac{4 \pi \mu_{S}}{L} W \equiv K_{2} W
$$

By (4.7d), this estimate gives

$$
k_{1}=2 \sqrt{\pi \frac{\mu_{S} h}{\bar{E} L}}
$$

For stiff films on highly compliant substrates, $\mu_{S} / \bar{E}<<1$ and $h / L<<1$; therefore, $k_{1}<<1$. For example, for typical stiff film/PDMS systems, $h / L \approx 1 / 10$ and $\mu_{S} / \bar{E}_{f} \approx 10^{-5}$ such that $k_{1} \approx 3 \times 10^{-3}$. Even in the mathematical limit, $k_{1}=0$, the tangential displacement $\Delta u$ has an important role, as will be seen.

The post-bifurcation modes of interest depend on the nature of the nonlinearity. There are no secondary bifurcations if the normal traction-displacement relation is linear. The nature of the nonlinearity of the normal traction-displacement relation in (4.1) and (4.7a) depends on whether the substrate is pre-stretched or pre-compressed, as has been 
discussed in Section 3.2. The example in Fig. 8 with $k_{22}=-0.5$ and $k_{23}=0.1$ represents the type of nonlinearity of a substrate with positive pre-stretch (compare with Fig. 7), and it will be used in the numerical simulations presented below.

At best, the nonlinear foundation model (4.1) can only approximate a continuum substrate such as a neo-Hookean half-space. For example, the traction-displacement relation of a continuum half-space depends on the wavelength of the deflection as seen in (4.9), while (4.1) has no such dependence. Nevertheless, (4.1) captures qualitative aspects of the nonlinearity and it can be calibrated to a particular wavelength.

\subsection{Primary solution}

In dimensionless variables, the eigenvalue, eigenmode and wavelength in (4.6) associated with the primary (lowest) bifurcation are

$$
e_{0}^{C}=1 / \sqrt{3}, w_{C}=\cos (2 \pi x / \ell), \Delta u=0, \ell \equiv L / d=2 \pi /(12)^{1 / 4}=3.376
$$

For loading beyond the critical bifurcation point with $e_{0}>e_{0}^{C}$, a periodic solution to the nonlinear system of equations (4.7) with wavelength $\ell$ emerges from (4.11) - this finite amplitude solution will be referred to as the primary solution. Numerical methods for solving nonlinear ode's, such as those available in IMSL (2009), are required to generate these solutions because it is not possible to solve the system (4.7) analytically. The primary solutions are readily generated; their symmetry with respect to the center of the period can be exploited. The primary solution is illustrated in Fig. 9. Of particular note are: (i) upward deflections favored over downward deflections due to the asymmetry in the normal traction-displacement relation (i.e., $k_{22}<0$ ); (ii) near-independence of $w$ on the tangential stiffness coefficient, $k_{1}$. In the limit $k_{1}=0$, (4.7b) implies that $n$ is independent of $x$. Nevertheless, the tangential displacement still has a role to play because, by (4.7c), periodicity of $u$ provides the relation of $n$ to prescribed $e_{0}$ as

$$
n=-e_{0}+\frac{1}{2 \ell} \int_{0}^{\ell} w^{\prime 2} d x
$$

\subsection{Secondary bifurcation solutions}


The next step in analyzing the model is to inquire whether secondary bifurcations from the primary solution occur as the system is loaded beyond primary bifurcation under increasing $e_{0}$. Here, the search for the secondary bifurcations has been limited to modes with periodicity $M \ell$ where $M$ is integer. With subscript “ $P$ ” denoting the primary solution, the linearized equations for the secondary bifurcation variables, $(\tilde{w}, \Delta \tilde{u}, \tilde{n})$, are

$$
\begin{aligned}
& \frac{1}{12} \tilde{w}^{\prime \prime \prime}-\left(n_{P} \tilde{w}^{\prime}\right)^{\prime}-\left(\tilde{n} w_{P}^{\prime}\right)^{\prime}+\tilde{w}+2 k_{22} w_{P} \tilde{w}+3 k_{23} w_{P}{ }^{2} \tilde{w}=0 \\
& \tilde{n}^{\prime}=k_{1} \Delta \tilde{u} \\
& \tilde{n}=\Delta \tilde{u}^{\prime}+w_{P}^{\prime} \tilde{w}^{\prime}
\end{aligned}
$$

subject to boundary conditions consistent with the period $M \ell$. Although linear, these equations have $x$-dependent, coefficients with period $\ell$. Solutions have been generated numerically using ode solvers in IMSL.

The lowest normalized eigenvalues, $e_{0} / e_{0}^{C}$, associated with secondary bifurcations are presented in Figs. 10. For the system with $k_{22}=-0.5$ and $k_{23}=0.1$, secondary bifurcations with $M=3$ occur at the lowest $e_{0}$, and these exist only for values of the dimensionless tangential stiffness coefficient, $k_{1}$, less than about 0.01 . For $k_{1}$ larger than about 0.01 , no secondary bifurcations have been found for any $M$. No secondary modes for $M=1$ were found for any $k_{1}$. For integer values $M>1$, secondary eigenvalues exist at larger values of $e_{0}$ than those plotted in Fig. 10, but these are of less interest. No secondary bifurcations were found when the normal traction-displacement relation is linear (i.e., $k_{22}=k_{23}=0$ ).

An example of a secondary bifurcation eigenmode is displayed in Figs. 11 for $M=5$ where the mode has been normalized such that the maximum value of $\tilde{w}$ is unity. The shape of the deflection is relatively insensitive to $k_{1}$, even though $k_{1}$ plays an strong role in determining whether or not secondary bifurcation occurs. The secondary modes have amplified undulation magnitude in some regions and diminished magnitude in others indicating the onset of localization.

\subsection{Fully nonlinear solutions illustrating ridge formation}


Solutions to the fully nonlinear system of ordinary differential equations (4.7) have been generated numerically using the ode solver DBVPFD in IMSL. The search has been restricted to periodic solutions with period $M \ell$, where $M$ is integer. It is not straightforward to obtain these solutions - patience and persistence are required. The primary solution with periodicity $\ell$ always exists for $e_{0}>e_{0}^{C}$ because it also has period $M \ell$. Additional solutions do not necessarily exist, as, for example, when $k_{1}>0.01$ or if $e_{0}$ is below all of the eigenvalues associated with secondary bifurcation. When other solutions do exist, one method which successfully produced them (with requisite trial and error) entailed the introduction of a small initial undulation imperfection into system (4.7) that encompassed a superposition of many possible mode shapes. A solution was generated using the ode solver with the imperfection present. Then, the parameter tracking option in the solver can be used to reduce the imperfection to zero in steps. If successful, the resulting solution is a converged solution to the fully nonlinear system (4.7). Once a solution has been obtained for one set of parameters, the parameter tracking option can be used to generate solutions for other sets of parameters.

The outcome of this process is shown in Figs. 12 and 13 for $M=6$. Fig. 12 displays the solution for three values of imposed overall compression, while Fig. 13 shows the effect of increasing the dimensionless tangential stiffness of the substrate. The period, $6 \ell$, of the solution is large enough to reveal aspects of ridge formation. The shape of the normal deflection is qualitatively similar to that seen in the experiments. Dominant ridge peaks with neighboring lesser peaks are separated by regions with small amplitude undulations.

Solutions with the general features of the fully nonlinear solutions presented here have been produced by Hunt et al. (1989) and Wadee et al. (1997) for the model system (4.7) for the special case with $k_{1}=0, k_{22} \neq 0$ and $k_{23}=0$. These authors also emphasize the localized deflection behavior and discuss various techniques for producing solutions to the nonlinear ordinary differential equation (4.7a). For the system with no tangential constraint of the substrate ( $k_{1}=0$ ), the single nonlinear equation (4.7a) is decoupled from (4.7b). The dimensionless horizontal force carried by the film, $n$, in the limit $k_{1}=0$, is independent of $x$ and becomes, in effect, the load parameter. As noted earlier, within the 
context of a film/substrate system, the tangential displacement still has a role to play in this limit: (4.12) gives $n$ in terms of the imposed overall compressive strain, $e_{0}$.

The solutions in Figs. 12 and 13 can be used to obtain a qualitative understanding of why such solutions do not exist if the dimensionless tangential stiffness, $k_{1}$, is too large. The contributions to the energy in (4.8) from the linear tangential and normal restoring tractions are $\left(k_{1} / 2\right) \int_{0}^{M l / d} \Delta u^{2} d x$ and $(1 / 2) \int_{0}^{M l / d} w^{2} d x$, respectively. For the examples in Figs. 12 and 13, the ratio of $\int_{0}^{M l / d} \Delta u^{2} d x$ to $\int_{0}^{M l / d} w^{2} d x$ is greater than 10 in all cases and relatively insensitive to $k_{1}$. Thus, the energy stored in the substrate due to the tangential displacement will be comparable to the energy stored due the normal displacement unless $10 k_{1}<<1$. Because the energy associated with the tangential displacement is parasitic, it will tend to suppress localization unless $10 k_{1}<<1$. The estimate, $k_{1} \approx 3 \times 10^{-3}$, made earlier for typical stiff films on PDMS substrates, which can localize, is consistent with this requirement, as is the fact that no ridge modes were found for the system investigated in this section if $k_{1}>0.01$.

The solutions in Fig. 12 and 13 also illustrate an important aspect of localization in the ridge mode. The tangential displacement in the central region where the undulations are small is directed towards the nearest ridge. The film must displace tangentially in this region to provide the extra film length required to form the ridge. As noted earlier, a ridge grows at the expense of its neighboring undulations, and the tangential displacement of the film is crucial to this localization process. When the tangential stiffness is too large, tangential film displacement is suppressed and localization cannot occur. Localization in the ridge mode is even more evident in the distribution of the energy density (i.e., the integrand in (4.8)) plotted in Fig. 14.

Wrinkling localization under compression seen for the film/substrate system is reminiscent of buckling localization that has been observed to occur when railroad tracks become over heated by the sun (Tvergaard and Needleman, 1981). Large lateral buckling deflections at one location along the tracks relieve compression in neighboring sections 
of the tracks rendering them less prone to buckle. In both systems, the nonlinearity of the substrate constraint plays a critical role in determining whether or not localization occurs.

\subsection{Fully nonlinear solutions illustrating fold formation}

A fold is a ridge of opposite sign — a localized undulation of the film that penetrates deeply into the substrate surrounded by undulations of smaller amplitude. The

substrate nonlinearity considered in the previous sections (with $k_{22}=-0.5$ and $k_{23}=0.1$ ) was chosen to give rise to softening behavior for outward normal deflections of the substrate surface and hardening behavior for inward deflection, qualitatively consistent with the effect of a pre-stretch seen in Fig. 7. If instead, the sign of $k_{22}$ is changed with all other parameters unchanged (i.e., $k_{22}=0.5$ and $k_{23}=0.1$ ) then softening occurs for inward deflections and hardening for outward deflections. These conditions favor the formation of folds.

For a given value of $k_{22}$, it is readily shown that any solution, $(w, u)$, to the fully nonlinear system (4.7) generates a solution, $(-w, u)$, to the system when the sign of $k_{22}$ is changed, with all other parameters unchanged. Thus, with due regard for the sign change of $w$, all the prior results for $\left(k_{22}=-0.5, k_{23}=0.1\right)$ apply to $\left(k_{22}=0.5, k_{23}=0.1\right)$. In particular, the fully nonlinear solutions in Fig. 12 and 13, with a sign change for $w$, can be interpreted as fold-like deformations.

\section{Conclusions}

The ridge localization mode in a film/substrate system under plane strain compression can take place when the substrate nonlinearity gives rise to a softer response for a local outward normal deflection than for an opposing inward deflection. Ridge formation also requires the tangential stiffness of the substrate to be sufficiently low such that the film can displace laterally allowing the ridge to grow at the expense of neighboring undulations. Pre-stretch of an elastomer substrate has been shown to generate nonlinearity favorable to localization and the formation of ridge localizations. The compression experiments in this paper on pre-stretched PDMS substrates with silica- 
like films formed by surface oxidation have revealed localized amplification of the undulations but not ridge formation. The occurrence of localization as dependent on the level of substrate pre-stretch is in general agreement mode with the simulations, as is the fact that the localization reverts back to the periodic sinusoidal-like mode as the compression is further increased. In particular, both our experiments and finite element simulations reveal that the PDMS substrate must be pre-stretched by more than about $40 \%$ for localization to be observed. The formation of localized ridges, or outward folds, has been noted in the experiments of Ebata, et al. (2012), but discrepancy between these observations and the present simulations exists in that the pre-stretch in the experiments was well below $40 \%$. Thus, a robust experimental confirmation of the ridge mode remains to be achieved.

Folds can form when the substrate nonlinearity favors inward over outward local normal deflections. For elastomeric substrates described by either neo-Hookean or Arruda-Boyce models the present simulations reveal that pre-compression of the substrate can create this type of nonlinearity. However, the form of the nonlinearity produced by pre-stretch and pre-compression are not mirror images of one another, as can be seen in Fig. 7. This may be the reason that the wrinkling sequence leading to folds differs from that for ridges. When the substrate has been pre-compressed (c.f. Fig. 6), the sinusoidal mode gives way to period-doubling with folds developing gradually with further applied compression. If the substrate has not been pre-deformed, the same sequence takes place but the sinusoidal mode is stable to relatively large applied compressive strains of about 0.2 before period-doubling and folding set in (Brau et al., 2010; Sun et al., 2012; Cao and Hutchinson, 2012a). Presumably, a compressive strain of around 0.2 is required to create the substrate nonlinearity required for formation of these advanced modes.

The differential equation model of the film/substrate system proposed in Section 4 enhances earlier models used to explore advanced modes by including the tangential restraint of the substrate on the film. The model reveals the important role played by this restraint on the advance modes. In particular, it shows that the localization aspect of the advanced modes is suppressed if the tangential stiffness exceeds a critical level. Onedimensional models such as this are mainly limited by the difficulty of accurately 
representing the nonlinearity of a substrate material, which is generally quite complex. Nevertheless, the models can provide important qualitative insights to the highly nonlinear wrinkling phenomena.

\section{Acknowledgements}

J.Z. and X.Z. acknowledge the supports from Duke Pratt School of Engineering and NSF's Research Triangle MRSEC (DMR-1121107), Y. P. C. acknowledges the supports from the National Natural Science Foundation of China (Grant No. 11172155) and Tsinghua University (2009THZ02122). 


\section{References}

ABAQUS. 2008. ABAQUS analysis user's manual, version 6.8.

Allen, H. G., Analysis and Design of Structural Sandwich Panels, Pergamon, New York, 1969.

Arruda, E. M. and Boyce, M. C., 1993. A three-dimensional model for the large sretch behavior of rubber elastic materials. J. Mech. Phys. Solids, 41, 389-412.

Befahy, S., Lipnik, P., Pardoen, T., Nascimento, C., Paris, B., Bertrand, P., Yunus, S., 2010. Thickness and elastic modulus of plasma treated PDMS Silica-like surface layer. Langmuir 26, 3372-3375.

Bowden, N., Brittain, S., Evans, A. G., Hutchinson J. W., and Whitesides, G. M., 1998. Spontaneous formation of ordered structures in thin films of metals supported on an elastomeric polymer. Nature 393, 146-149.

Brau, F., Vandeparre, H., Sabbah, A., Poulard, C., Boudaoud, A., and Damman, P., 2010. Multiple-length-scale elastic instability mimics parametric resonance of nonlinear oscillators, Nat. Phys. 7, 56-60.

Cai, S., Breid, D., Crosby, A.J., Suo, Z., Hutchinson, J.W., 2011. Periodic patterns and energy states of buckled films on compliant substrates. J. Mech. Phys. Solids, 59, 10941114.

Cao, Y and Hutchinson, J. W., 2012a, Wrinkling phenomena in neo-Hookean film/substrate systems, to be published in J. Appl. Mech.

Cao, Y., Hutchinson, J. W., 2012b, From wrinkles to creases in elastomers: the instability and imperfection-sensitivity of wrinkling. Proc. R. Soc. A. 468, 94-115.

Ebata, Y., Croll, A.B., Crosby, A.J., 2012. Wrinkling and strain localizations in polymer thin films. (submitted for publication).

Groenewold, J., 2001. Wrinkling of plates coupled with soft elastic media. Physica A 298, p.32-45.

Huang, R., Suo, Z., 2002. Instability of a compressed elastic film on a viscous layer. Int. J. Solids Struct. 39 (7), 1791-1802.

Huang, R., 2005. Kinetic wrinkling of an elastic film on a viscoelastic substrate. J. Mech. Phys. Solids 53 (1), 63-89. 
Huang, Z.Y., Hong, W., Suo, Z., 2005. Nonlinear analyses of wrinkles in a film bonded to a compliant substrate. J. Mech. Phys. Solids 53 (9), 2101-2118.

Hunt, G. W., Bolt, H. M., Thompson, J. M. T., 1989. Structural localization phenomena and the dynamical phase-space analogy. Proc. R. Soc. Lond. A 1989 425, 245-267.

IMSL, 2009. Fortran Numerical Library, Version 5.0, Visual Numerics Corp.

Khang, D. Y., Jiang, H. Q., Huang, Y., Rogers, J. A., 2006. A stretchable form of singlecrystal silicon for high-performance electronics on rubber substrates. Science 311, 208212.

Kim, P., Abkarian, M., Stone, H. A., 2011. Hierarchical folding of elastic membranes under biaxial compressive stress. Nat. Mater. 10, 952-957.

Lacour, S. P., Jones, J., Wagner, S., Li, T. and Suo, Z., 2005. Stretchable interconnects for elastic electronic surfaces. Proc. IEEE 93, 1459-1467.

Stafford, C. M. et al., 2004. A buckling-based metrology for measuring the elastic moduli of polymeric thin films. Na. Mater. 3, 545-550.

Sun, J-Y., Xia, S., Moon, M-Y., Oh, K.H., and Kim, K-S., 2012, “Folding wrinkles of a thin stiff layer on a soft substrate”, Proc. Roy. Soc. A doi 10.1098/rspa.2011.0567.

Tikhonov, A. N. \& Arsenin, V. Y., 1977. Solutions of ill-posed problems. New York, NY: John Wiley \& Sons.

Tvergaard, V., Needleman, A., 1981. On localized thermal track buckling. Int. J. Mech. Sci. 23, 577-581.

Volynskii, A.L., Bazhenov, S., Lebedeva, O.V., Bakeev N.F., 2000. Mechanical buckling instability of thin coatings deposited on soft polymer substrates. J. Mater. Sci. 35, 547-554.

Wadee, M. K., Hunt, G. W., Whiting, A. I. M., 1997. Asymptotic and Rayleigh-Ritz routes to localized buckling solutions in an elastic instability problem. Proc. R. Soc. Lond. A 453, 2085-2107. 


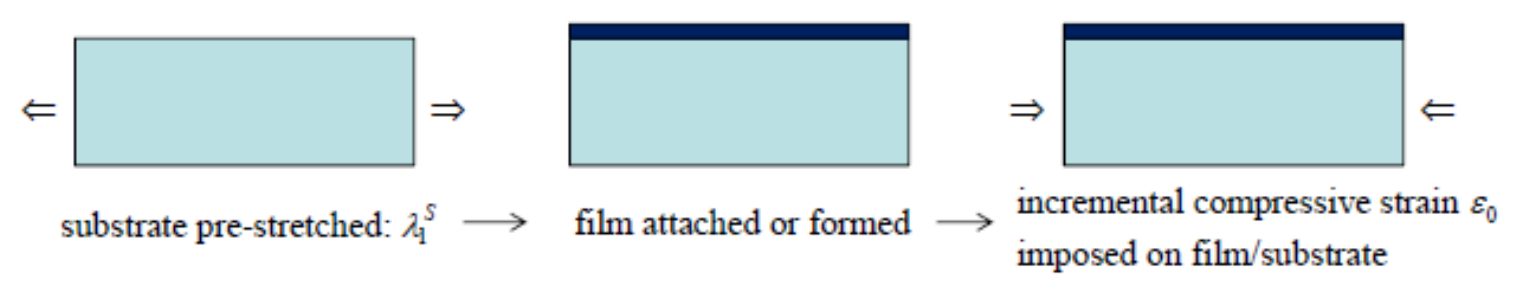

a)

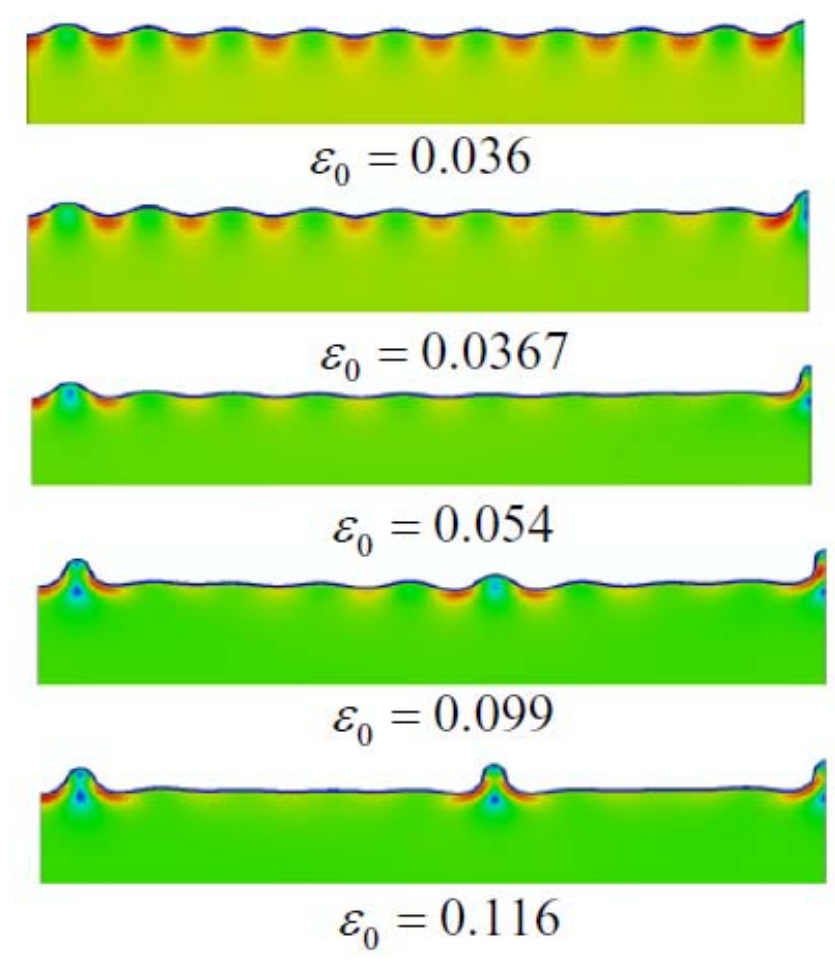

b)

Fig. 1 a) Schematic of loading sequence. b) Formation of localized ridges in a compressed stiff film/compliant system. Evolution of the wrinkling mode under plane strain compression, $\varepsilon_{0}$, for a neo-Hookean film attached to a neo-Hookean substrate following a plane strain pre-stretch $\lambda_{1}^{S}=2$ of the substrate, as simulated by Cao and Hutchinson (2012a). The film to substrate ground state shear modulus ratio is $\mu_{f} / \mu_{s}=836$. The sinusoidal wrinkling mode associated with bifurcation at $\varepsilon_{0}^{C}=0.01$ is stable to a compressive strain $\varepsilon_{0}=0.036$, but at $\varepsilon_{0}=0.0367$ a mountain ridge has formed at the right end of the model and the amplitudes of the undulations near the ridge have been reduced. By $\varepsilon_{0}=0.054$ a second ridge is clearly forming near the left end, and by $\varepsilon_{0}=0.099$ this ridge is fully developed with a third ridge beginning to emerge near the center. At $\varepsilon_{0}=0.116$ three fully developed ridges have formed and have relaxed the undulation amplitudes between the ridges. 
Fig. 2-Tensile stress-strain data of the PDMS used in the present experiments and the tensile predictions for the neo-Hookean model and the Arruda-Boyce model. The two constitutive models are taken to be incompressible and have been fit to a ground state shear modulus, $0.32 \mathrm{MPa}$. The locking parameter of the Arruda-Boyce model has been taken as $\lambda_{m}=1.17$. 

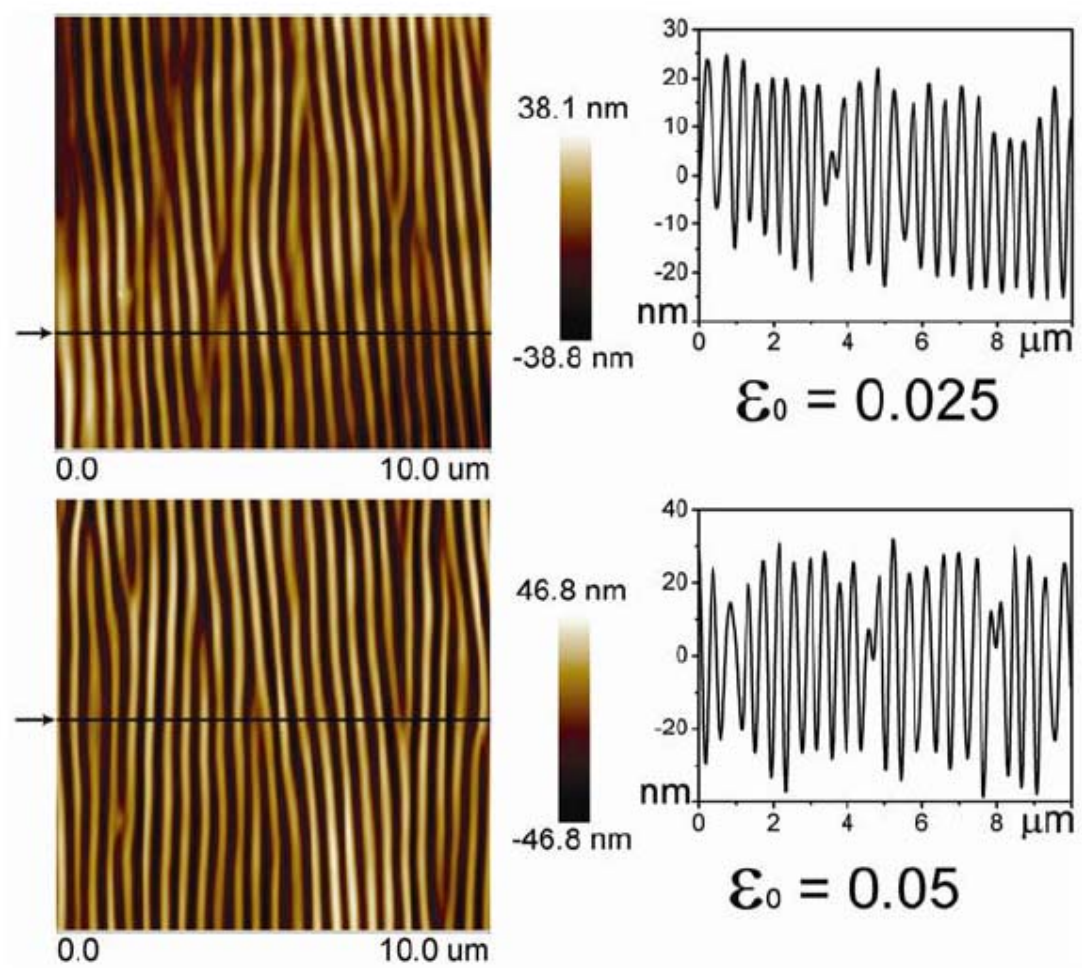

Fig. 3a Pre-stretch $\lambda_{1}^{S}=1.20$
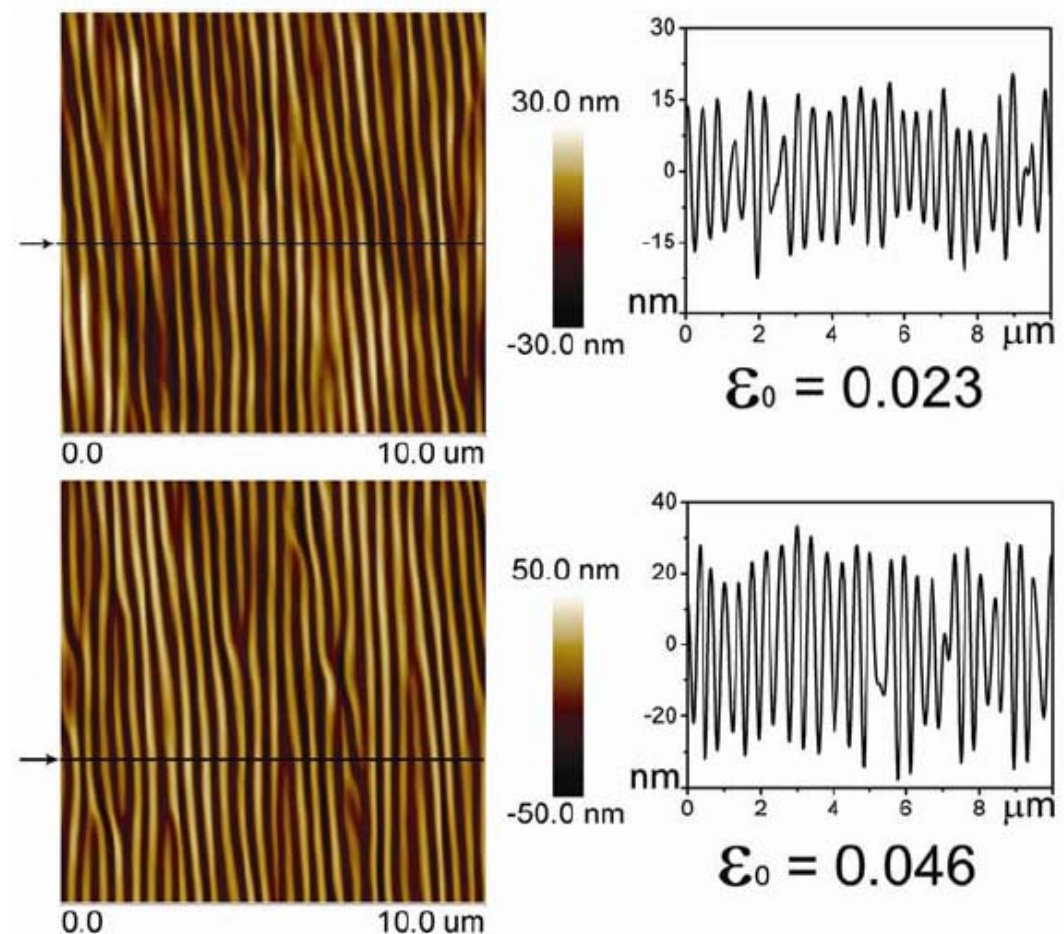

Fig. 3b Pre-stretch $\lambda_{1}^{S}=1.30$ 

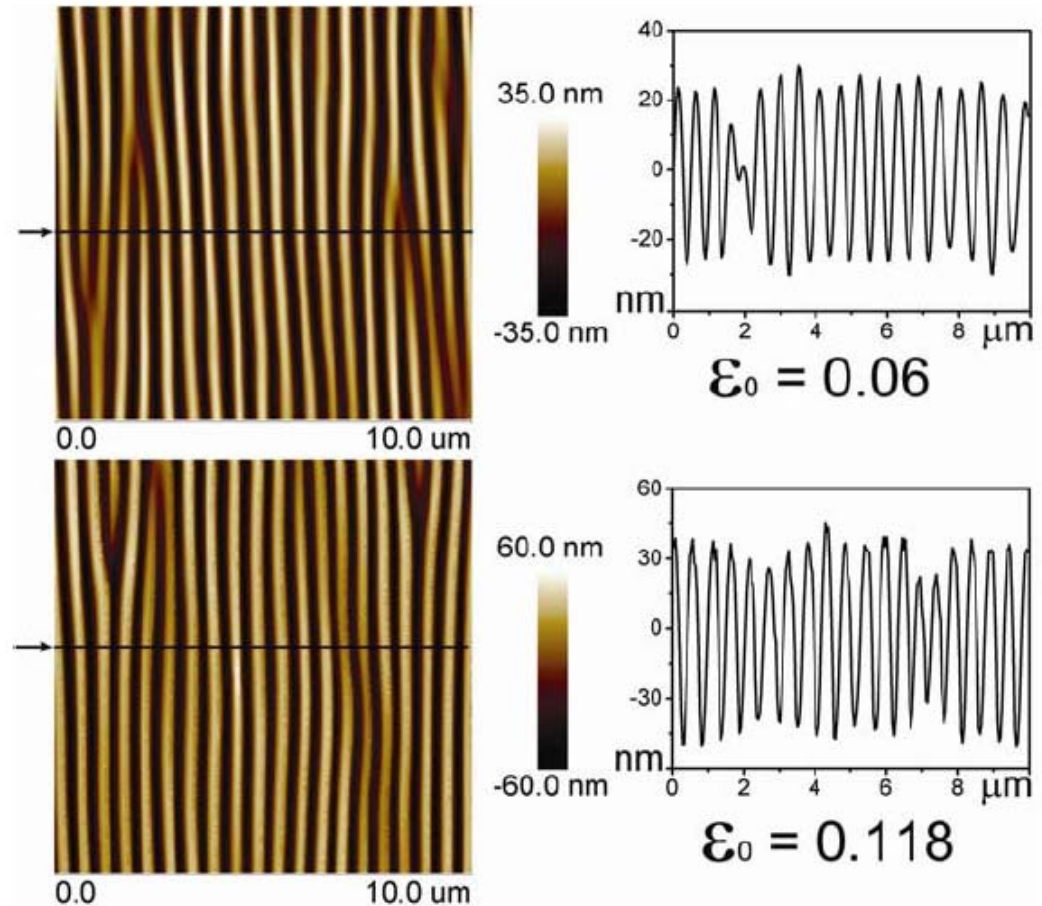

Fig. 3c Pre-stretch $\lambda_{1}^{S}=1.35$
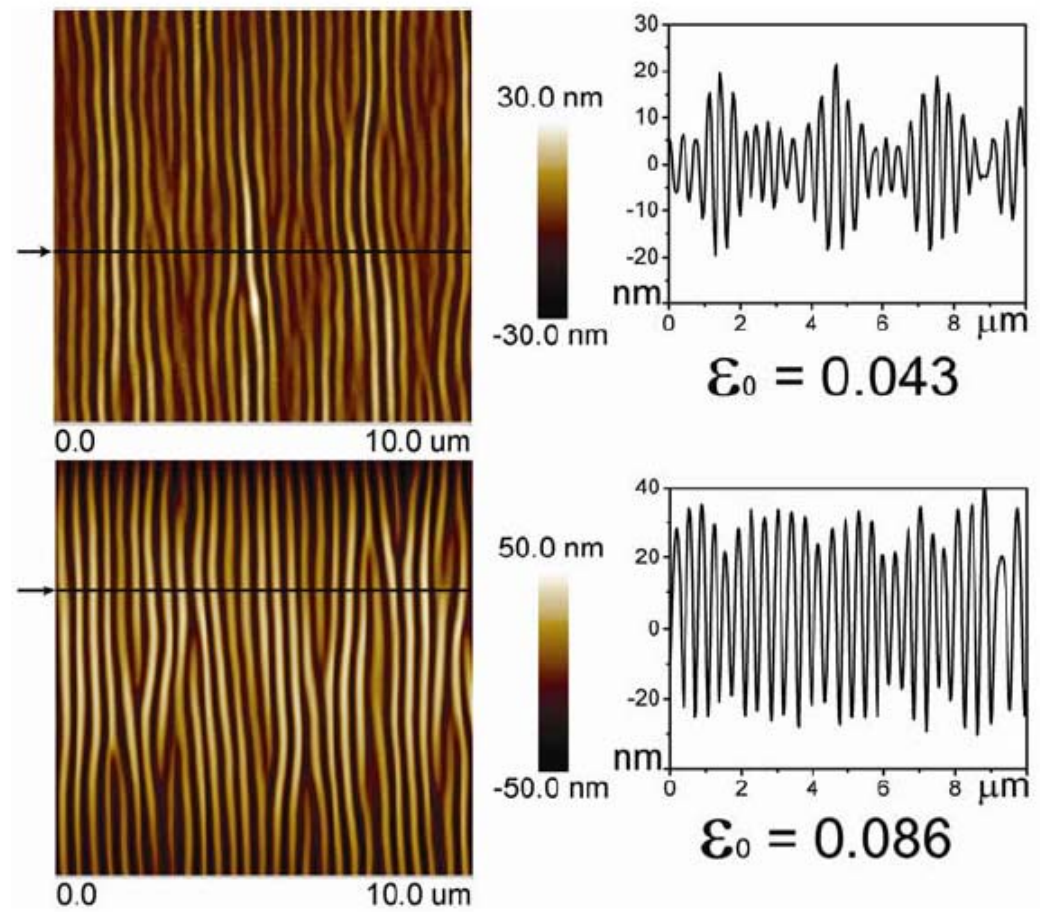

Fig. 3d Pre-stretch $\lambda_{1}^{S}=1.40$ 

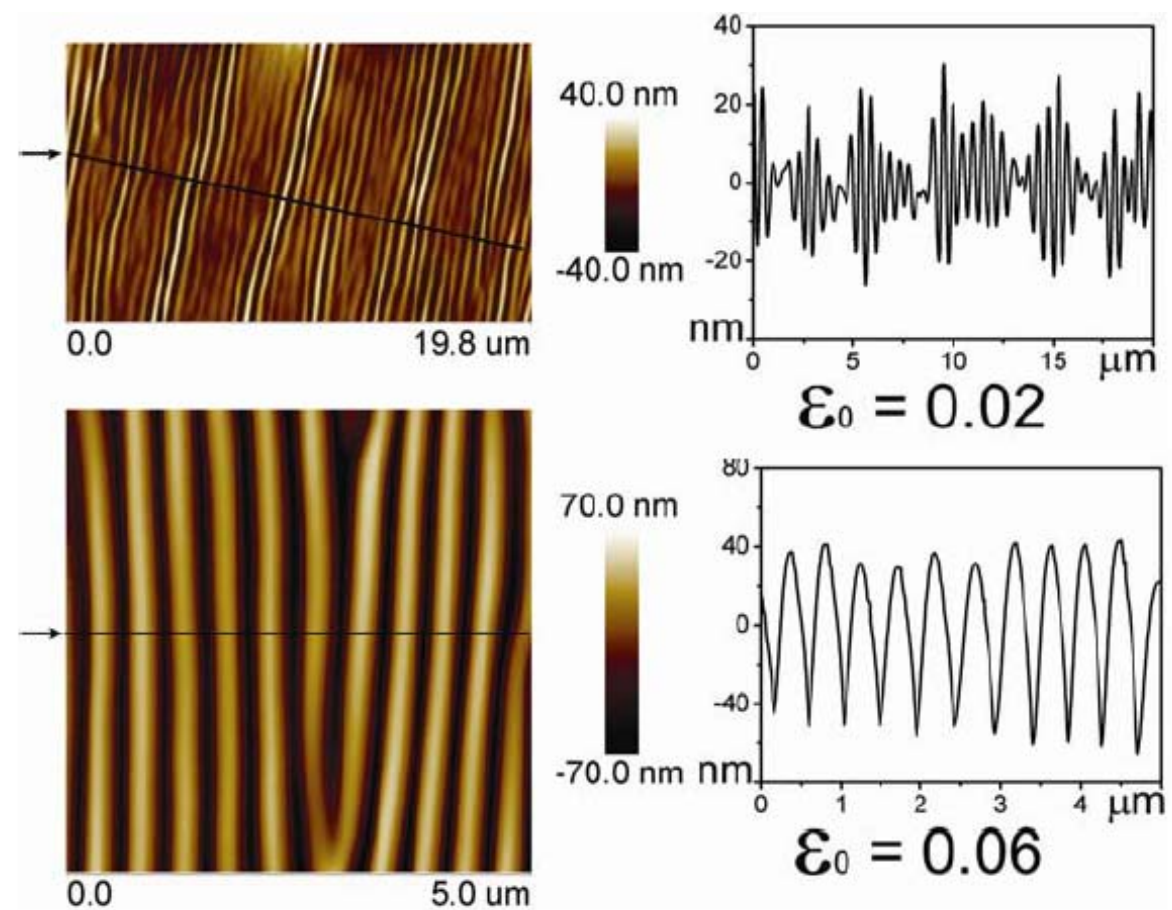

Fig. 3e Pre-stretch $\lambda_{1}^{S}=1.50$

Fig. 3 Experimental observations of wrinking of a stiff silica-like film formed by oxidation of the surface of a pre-stretched PDMS substrate. Five levels of plane strain pre-stretch, $\lambda_{1}^{S}$, are shown. The nominal compressive strain, $\varepsilon_{0}$, is imposed as a plane strain deformation on the pre-stretched system. Undulation localization is seen for $\lambda_{1}^{S}=1.35$ but only becomes well developed for $\lambda_{1}^{S}=1.40$ and $\lambda_{1}^{S}=1.50$. Note that the localization reverts back to a periodic mode as the compression increases to larger values. 

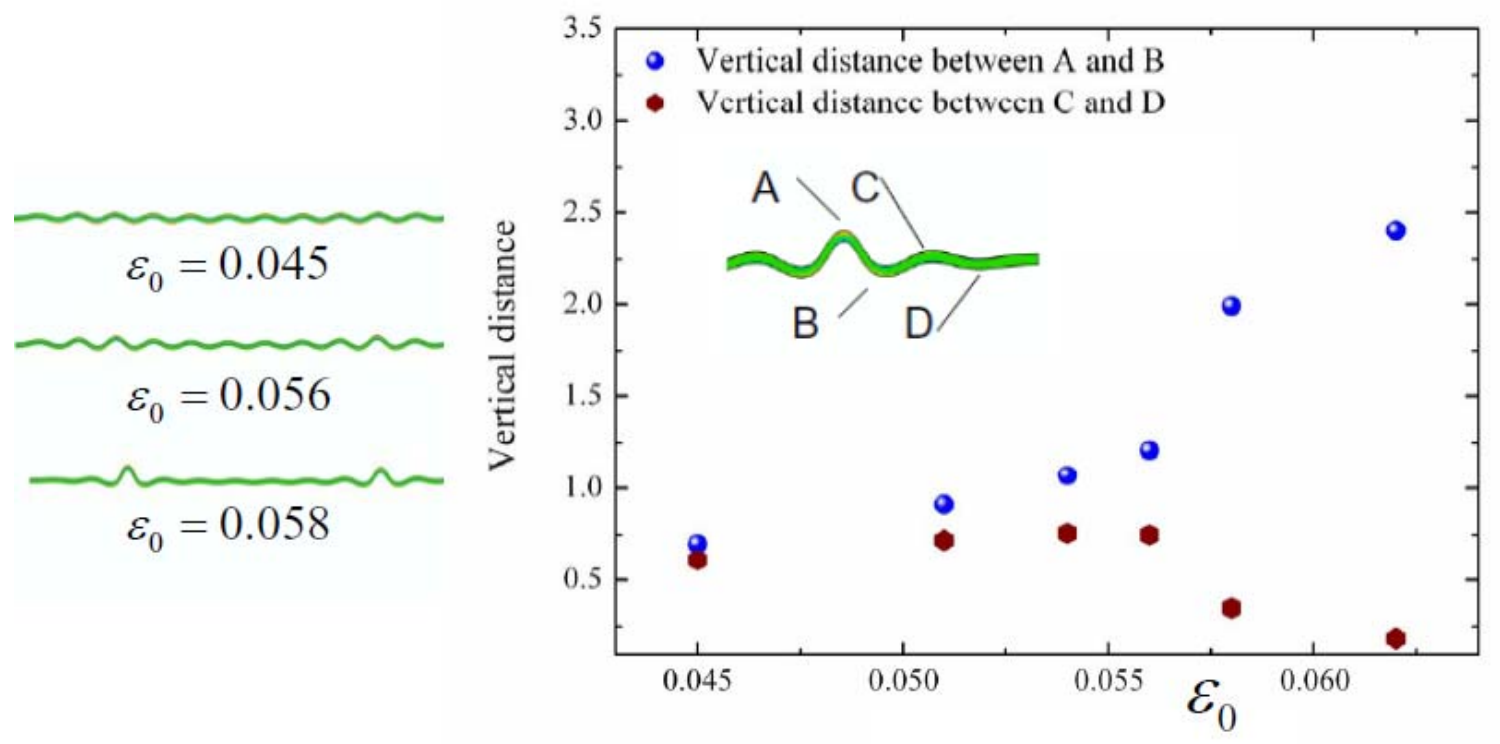

Fig. 4 The localization process is illustrated by the sharp increase in height of a ridge and the corresponding decrease in height experienced by the neighboring undulation.

Localization occurs over a small increase of compressive strain from about $\varepsilon_{0}=0.055$ to $\varepsilon_{0}=0.060$. This example is for a neo-Hookean film/substrate system with $\lambda_{1}^{S}=2$ and $\mu_{f} / \mu_{s}=123$. 


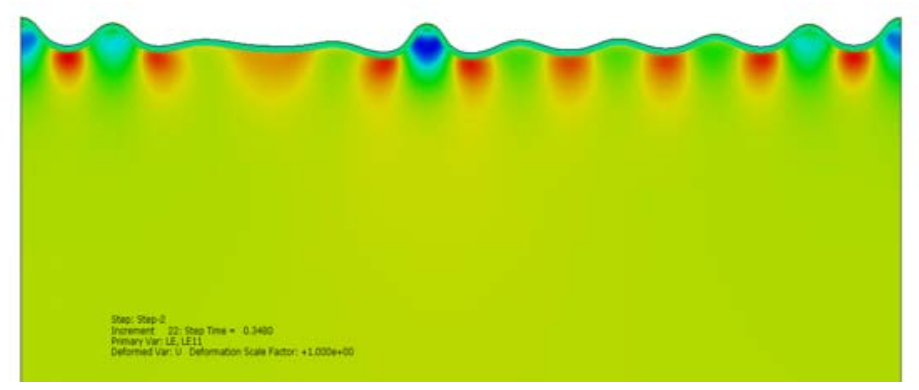

(a). Overall strain $\varepsilon_{0}=0.115$

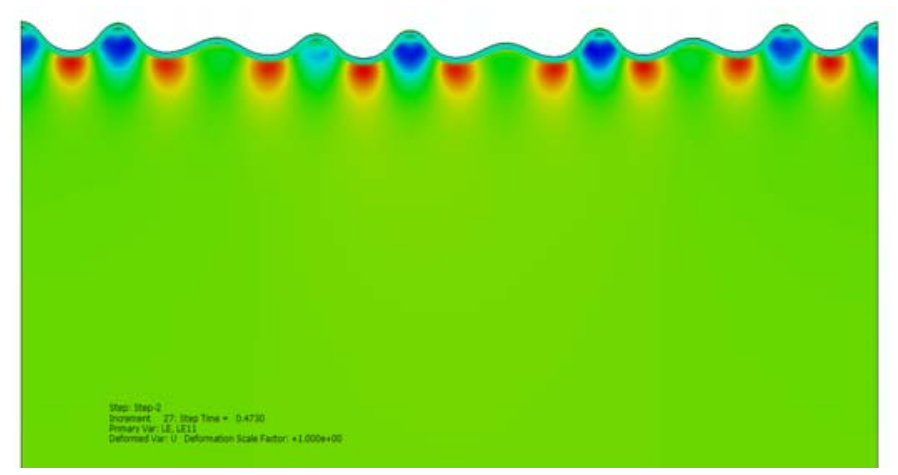

(b) $\varepsilon_{0}=0.16$

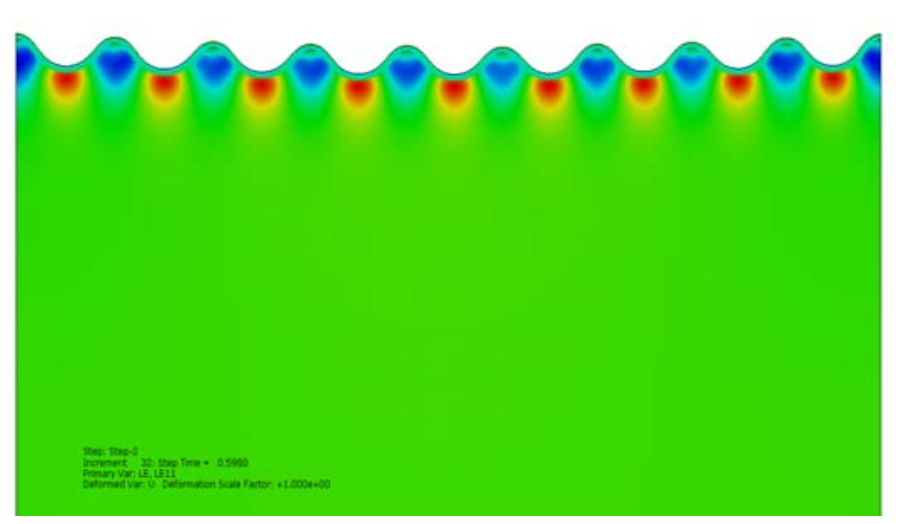

(c). $\varepsilon_{0}=0.20$

Fig. 5 Sequence of wrinkle patterns for an Arruda-Boyce film/substrate system with $\mu_{f} / \mu_{S}=100$ and a substrate pre-stretch $\lambda_{1}^{S}=1.5$. The simulation reveals that the ridge mode reverts back to the periodic sinusoidal mode as the compression is increased. Both the film and substrate are taken to be incompressible with locking parameter $\lambda_{m}=1.17$. 


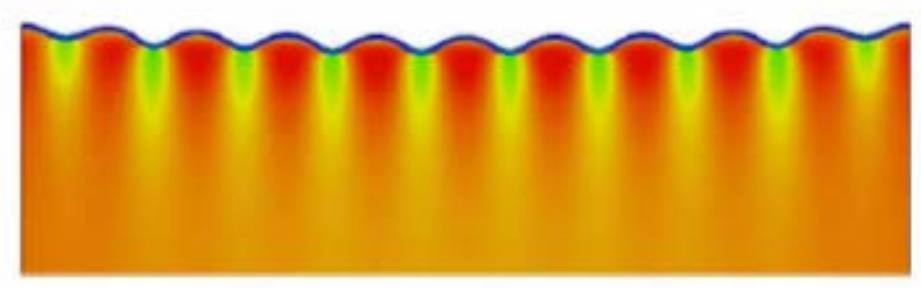

$$
\varepsilon_{0}=0.09
$$

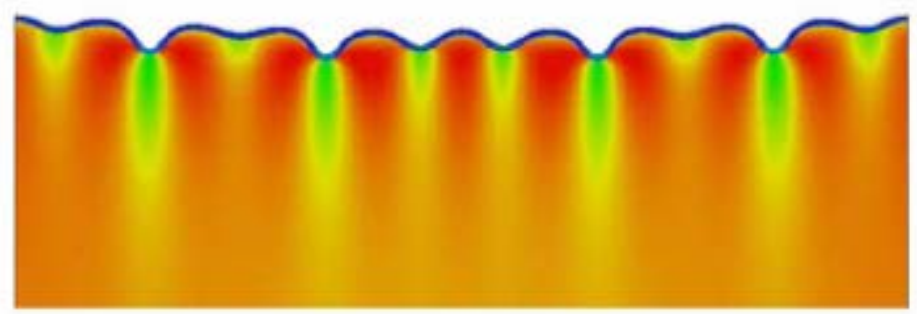

$$
\varepsilon_{0}=0.12
$$

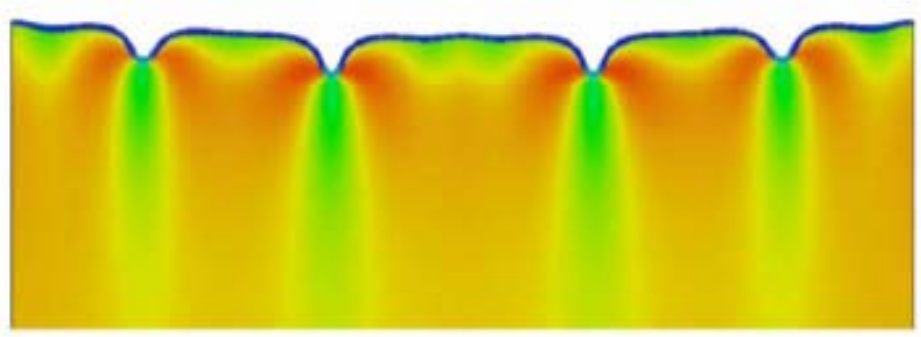

$$
\varepsilon_{0}=0.14
$$

Fig. 6 A sequence of wrinkle patterns showing the emergence of period-doubling followed by developing folds. The film and the substrate are modeled by the ArrudaBoyce model with the substrate fit to the data in Fig. 2 for PDMS. The film/substrate modulus ratio is $\mu_{f} / \mu_{s}=100$. The substrate is pre-compressed to $\lambda_{1}^{S}=0.7$ prior to attachment of the film and subsequent to imposition of the overall compressive strain $\varepsilon_{0}$. 


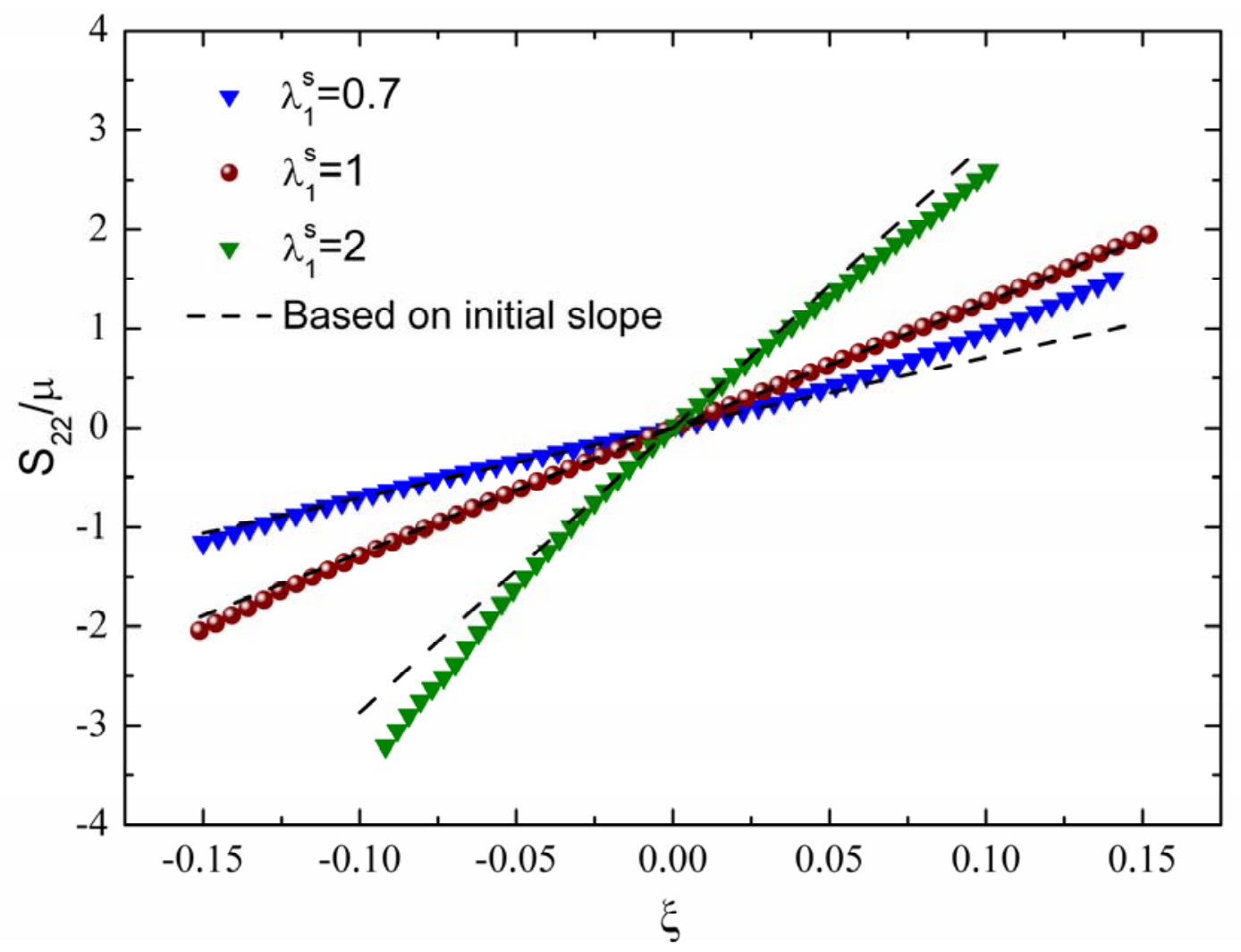

Fig. 7 Nonlinear normal traction-displacement relations for a semi-infinite neo-Hookean half-space subject to (i) no pre-stretch, $\lambda_{1}^{S}=1$, (ii) pre-stretch, $\lambda_{1}^{S}=2$, and (iii) precompression, $\lambda_{1}^{S}=0.7$. Following the uniform pre-deformation, nominal tractions, $\left(s_{22}, s_{21}\right)$, are imposed on the surface with $s_{21}=0$ and $s_{22}=p_{0} \cos (2 \pi X / L)$ where $X$ is the horizontal coordinate in the pre-deformed state. The normalized stress (force/predeformed area), $s_{22} / \mu_{s}$, at $X=0$ is plotted against $\xi \equiv u_{2} / L$ at $X=0$. For each case, the linearized response based on the initial slope is shown as a dashed line. 


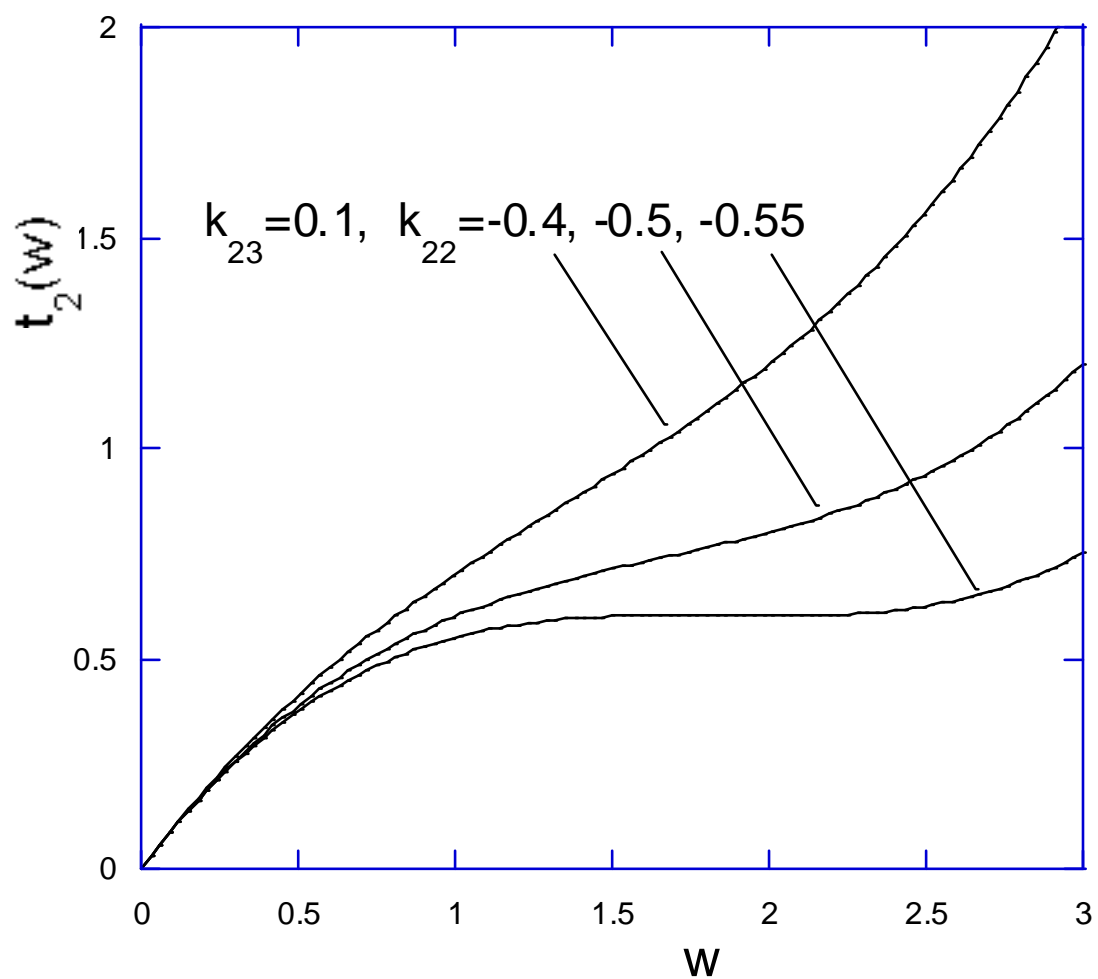

Fig. 8 An illustration of the dimensionless nonlinear normal traction-displacement relation with nonlinearity qualitatively representative of a substrate that has experienced pre-stretch (compare with Fig. 7). The curve with $k_{22}=-0.5$ and $k_{23}=0.1$ will be used in the simulations based on the one-dimensional model. 

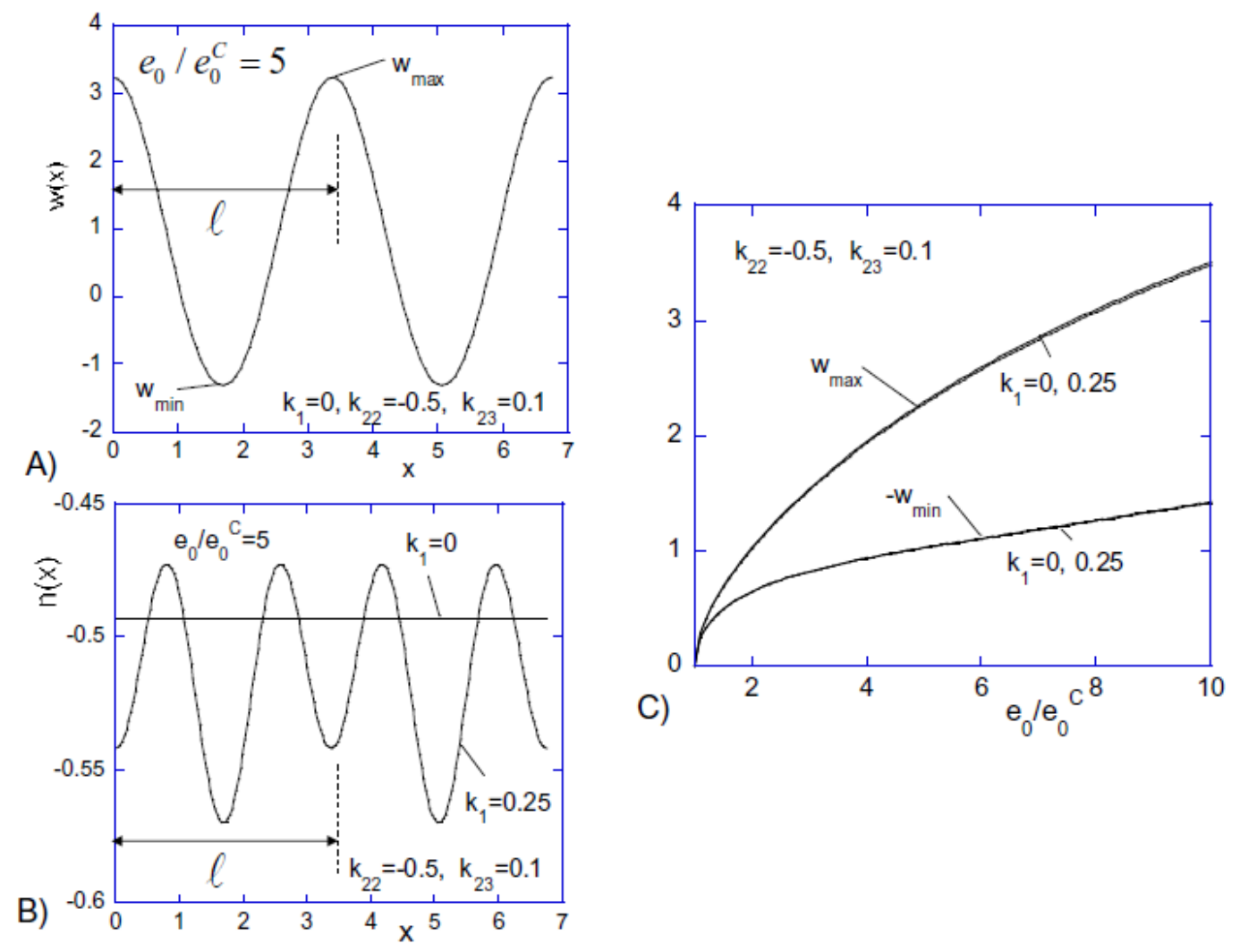

Fig. 9 The primary solution for $k_{1}=0$ and $k_{1}=0.25$ with $k_{22}=-0.5, k_{23}=0.1$. The normal displacement of the film, $w$, is almost independent of $k_{1}$. 


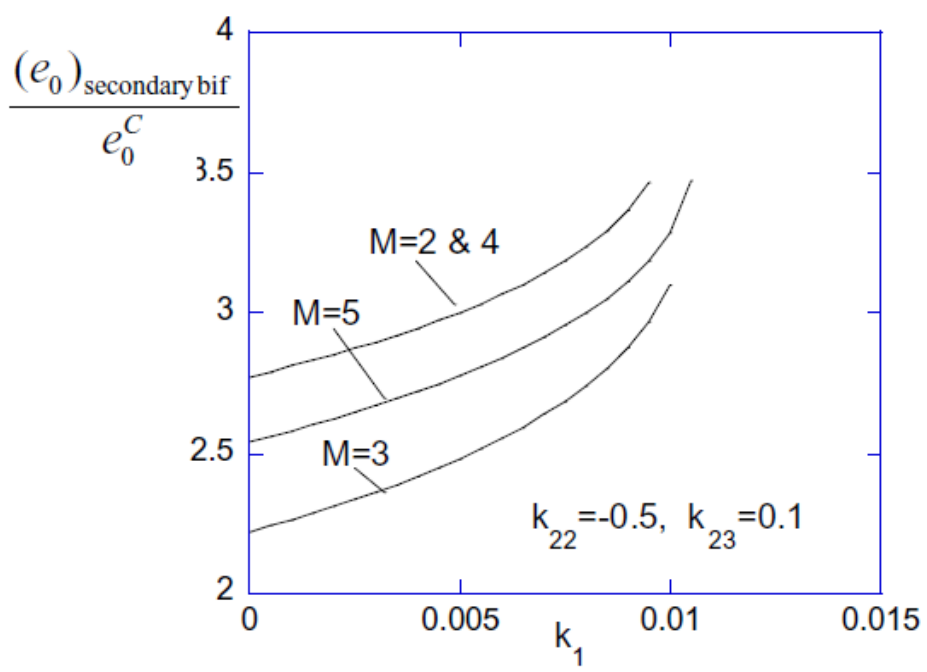

Fig. 10 The overall compressive strain associated with secondary bifurcation from the primary solution for secondary modes with periodic wavelengths, $M \ell$. The lowest strain for secondary bifurcation is associated with modes having $M=3$. An example of a secondary mode shape is given in Fig. 11. Secondary bifurcations with periodicities that are integer multiples of $\ell$ do not occur for $k_{1}$ greater than about 0.01 . 

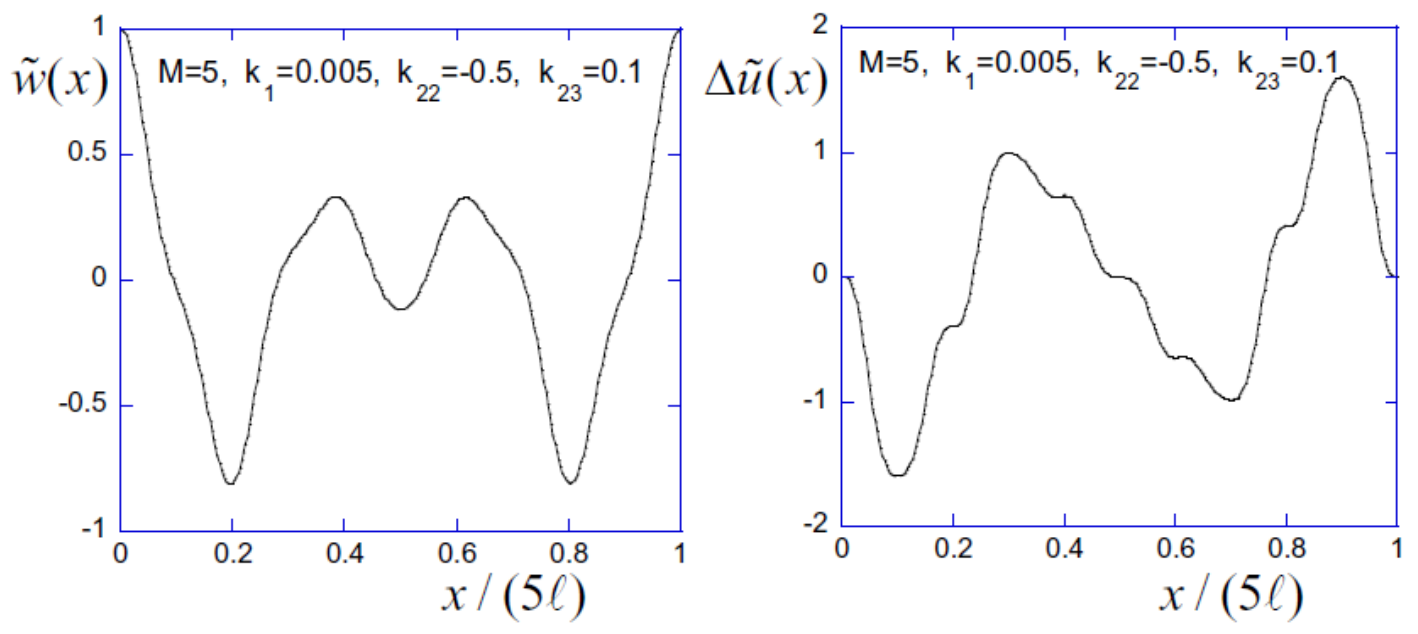

Fig. 11. Secondary bifurcation mode for $M=5, k_{1}=0.005, e_{0} / e_{0}^{C}=2.775$. 

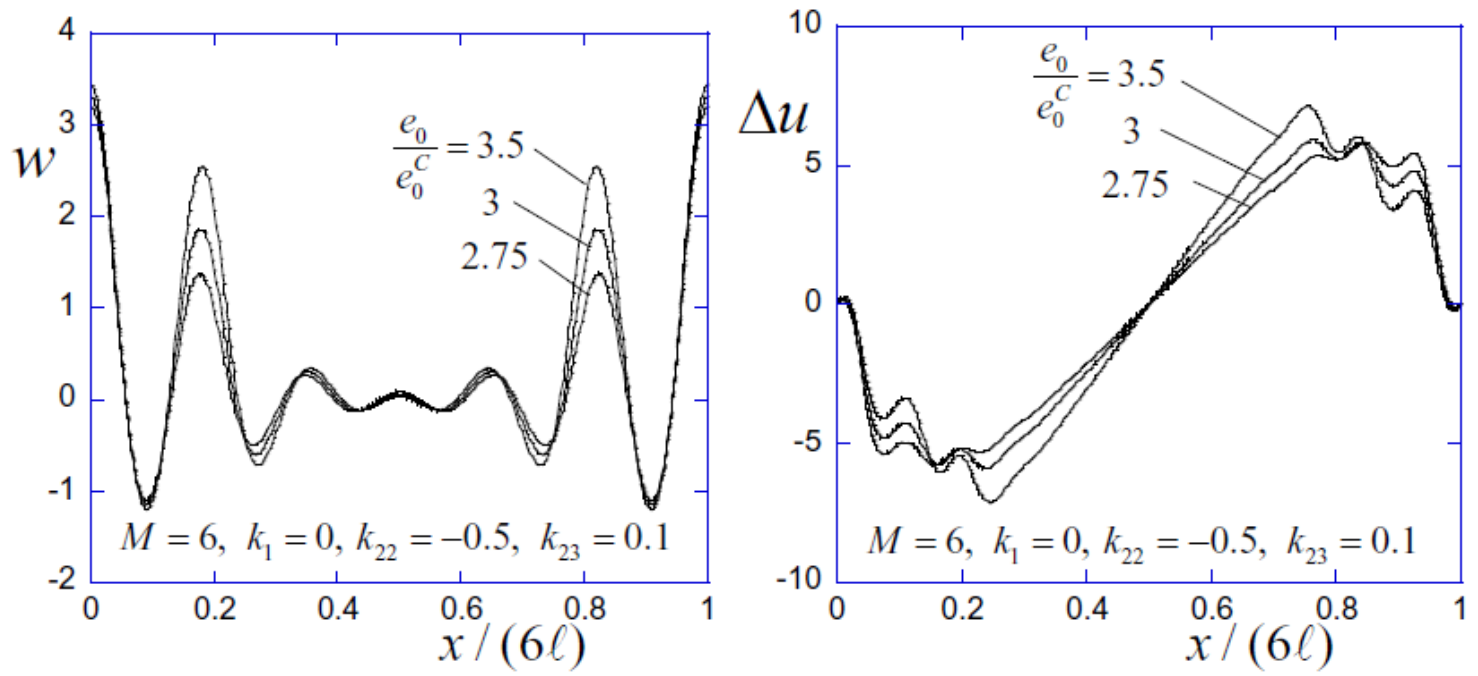

Fig. 12 Fully nonlinear solutions to the model for $M=6$ for several imposed overall strains, $e_{0}$. 

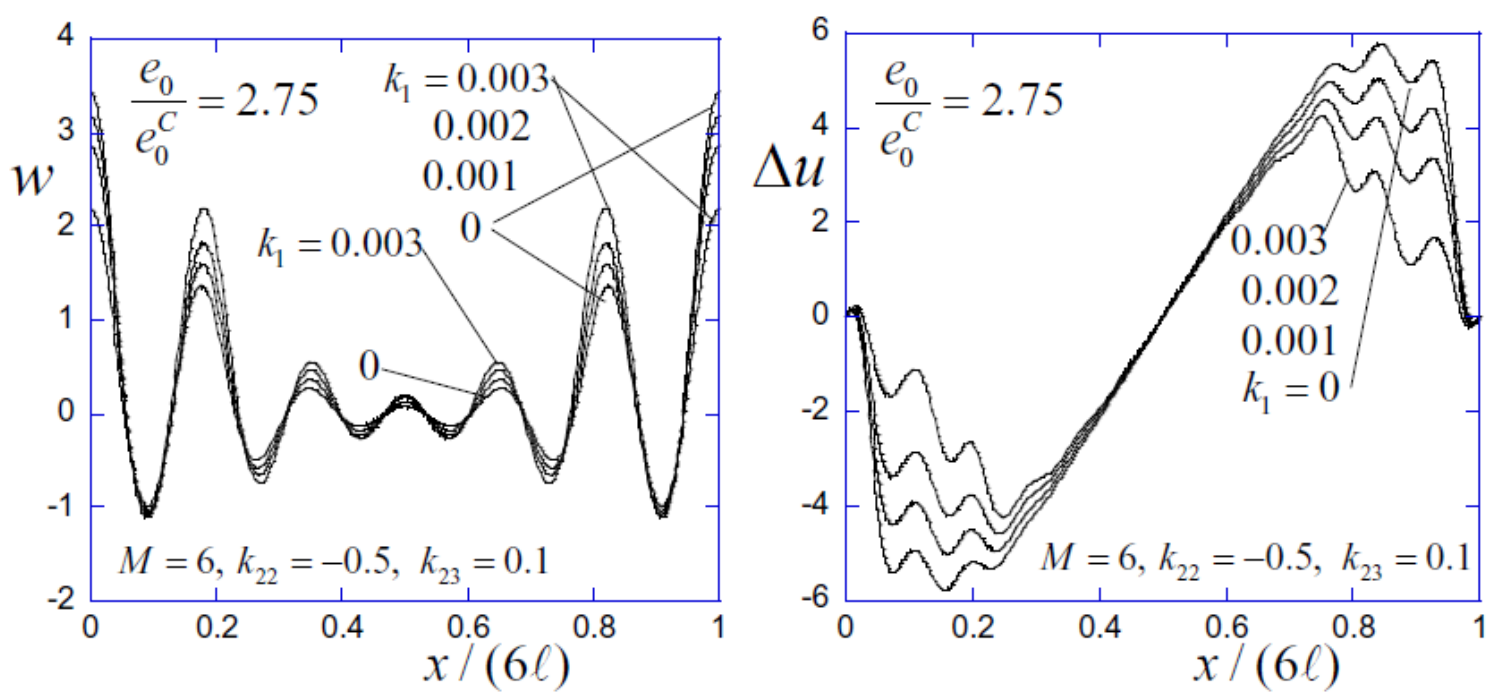

Fig. 13 Fully nonlinear solutions to the model for $M=6$ for several values of the dimensionless tangential stiffness, $k_{1}$. 


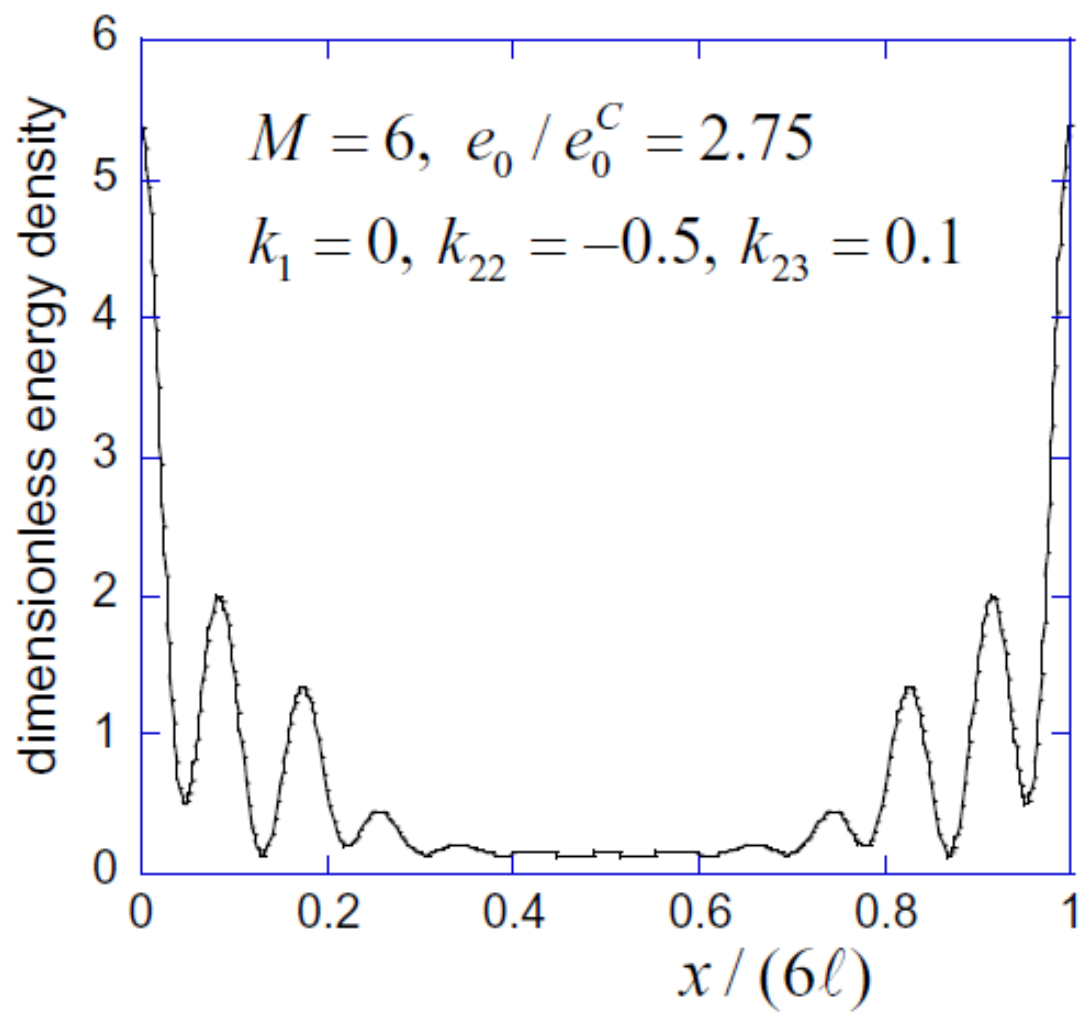

Fig. 14 Distribution of the dimensionless energy density of the film/substrate system (the integrand of (4.8)) for one of the fully nonlinear solutions in Fig. 12. This plot reveals the highly localized nature of the deformation in the vicinity of the ridges. 